\title{
Corela
}

Cognition, représentation, langage

HS-21 | 2017

Linguistique de corpus : vues sur la constitution, l'analyse et l'outillage

\section{Lier les cooccurrences des Epithetes (1571) de Maurice de La Porte et celles des textes de la Pléiade}

Nathalie Gasiglia et Anne-Pascale Pouey-Mounou

\section{CpenEdition}

Journals

Édition électronique

URL : https://journals.openedition.org/corela/4830

DOI : $10.4000 /$ corela. 4830

ISSN : 1638-573X

Éditeur

Université de Poitiers

Référence électronique

Nathalie Gasiglia et Anne-Pascale Pouey-Mounou, «Lier les cooccurrences des Epithetes (1571) de Maurice de La Porte et celles des textes de la Pléiade », Corela [En ligne], HS-21 | 2017, mis en ligne le 16 février 2017, consulté le 28 juin 2022. URL : http://journals.openedition.org/corela/4830 ; DOI : https://doi.org/10.4000/corela.4830

Ce document a été généré automatiquement le 29 septembre 2020

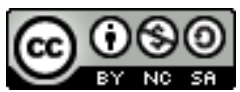

Corela - cognition, représentation, langage est mis à disposition selon les termes de la licence Creative Commons Attribution - Pas d'Utilisation Commerciale - Partage dans les Mêmes Conditions 4.0 International. 


\title{
Lier les cooccurrences des Epithetes (1571) de Maurice de La Porte et celles des textes de la Pléiade ${ }^{1}$
}

\author{
Nathalie Gasiglia et Anne-Pascale Pouey-Mounou
}

\section{Introduction}

Quels sont les choix méthodologiques spécifiques qu'implique le traitement d'un corpus lexicographique ancien, stylistiquement exploitable au sein d'un projet éditorial, et quel est l'apport d'une telle expérience? Les réflexions que nous présentons ici visent à souligner les contraintes et les solutions originales appelées par ce type d'objet, à partir d'un projet éditorial envisagé comme un cas d'école : le traitement et l'édition numérique en cours d'un recueil lexicographique qui eut un grand succès en son temps, Les Epithetes de Maurice de La Porte (1571)², premier dictionnaire d'épithètes en langue française. Pour le réaliser, son auteur a procédé à un relevé méthodique de cooccurrences de substantifs et d'épithètes (simples ou polylexicales) au sein des textes de la Pléiade, en particulier ceux de Ronsard" ${ }^{3}$. Son "épithétaire », pour reprendre le néologisme introduit par le lexicographe ${ }^{4}$, consiste en entrées nominales accompagnées d'une liste d'épithètes et éventuellement de commentaires.

Dans la double perspective d'une édition scientifique imprimée de cet ouvrage et, plus ambitieusement, d'une édition électronique enrichie de celui-ci, notre objectif est de repérer au sein des textes de la Pléiade quelles sont les attestations à partir desquelles La Porte peut vraisemblablement avoir effectué ses sélections. Alors que l'édition sur papier a vocation à ne fournir que des attestations minutieusement choisies des cooccurrences relevées par La Porte, une version électronique pourra en présenter de bien plus nombreuses, en spécifiant, à terme, quand cela peut être connu ou spéculé, si chacune est une source plausible pour La Porte ou s'il s'agit d'un réemploi ou encore d'un usage indépendant, voire si l'un des cooccurrents attestés s'est vu remplacer par un autre dans le texte dictionnairique. La base textuelle ainsi constituée contiendra donc le texte 
original de La Porte, enrichi de contextes issus de textes de la Pléiade dans lesquels les cooccurrences inventoriées par le lexicographe ont été observées. Elle sera proposée à la consultation sous la forme d'une version électronique du dictionnaire enrichie de citations, et dotée de fonctions de recherche permettant d'exploiter les informations bibliographiques (pour pouvoir sélectionner des sous-ensembles d'auteurs ou de textes) ou stylistiques (pour filtrer les contextes en fonction de la nature des épithètes, de leur valeur rhétorique, etc., cf. $\$ \S 2.1$ et 3.2.1.). L'élaboration de cette édition électronique maximalement enrichie se fera en deux temps : la première version, destinée à une étude plus approfondie comme à des consultations de curiosité, compilera les attestations repérées et permettra d'en faire des analyses fines, en les comparant entre elles; la seconde version, enrichie de données issues de l'analyse des cooccurrences, sera un objet éditorial dont le contenu sera stabilisé (avec ses attestations repérées et analysées) et que l'on pourra consulter isolément ou, éventuellement, exploiter en l'interfaçant avec d'autres corpus textuels électroniques pour permettre de comparer les cooccurrences de ces derniers et celles relevées par La Porte et enrichies des attestations que nous aurons collectées.

À l'égard des procédures habituelles de la linguistique de corpus, il nous faut souligner ici la triple singularité de ce projet. Elle tient d'abord à l'ancienneté du corpus envisagé, et aux difficultés d'accès et de traitement qu'entraînent spécifiquement les textes $\mathrm{du} \mathrm{XVI}^{\mathrm{e}}$ siècle. Elle est également liée aux compétences stylistiques, et non uniquement morphosyntaxiques, qu'impliquent le repérage et l'interprétation du phénomène épithétique, considéré à l'époque comme un fait rhétorique et non grammatical (cf. $\S 1.2$.). Elle résulte, enfin, du fait que la base textuelle en cours d'élaboration se veut à la fois un outil d'analyse et l'état préparatoire d'une édition numérique, dotée de fonctionnalités de recherche originales fondées sur l'annotation des attestations dont le texte de La Porte aura été enrichi.

Nous nous attacherons donc ici aux choix méthodologiques qu'implique cette spécificité de notre travail. Nous commencerons par situer Les Epithetes, en tant que dictionnaire de cooccurrences, par rapport à la production lexicographique actuelle et dans celle de son époque (cf. $§ 1$.$) . Nous présenterons ensuite notre projet d'édition électronique enrichie$ et nous développerons les contraintes que présentent la constitution et l'exploration d'un corpus de textes $\mathrm{du} \mathrm{xVI}^{\mathrm{e}}$ siècle où le repérage des cooccurrences lexicales impliquant des épithètes va régulièrement de pair avec la détection de faits rhétoriques ( $\$ 2$.$) . Nous$ reviendrons enfin sur les dépouillements effectués à ce jour, afin d'en exposer les objectifs, les conditions et les limites, ce qui nous fournira des éléments concrets pour présenter le potentiel informationnel des textes explorés et les stratégies manipulatoires qu'ils imposent pour leur rapprochement avec les cooccurrences relevées dans l'épithétaire (\$3.).

\section{Place relative du recours à la cooccurrence dans Les Epithetes et les dictionnaires du $\mathrm{XVl} \mathrm{l}^{\mathrm{e}}$ siècle ou actuels, généraux ou spécialisés}

Afin d'expliciter les enjeux atypiques des manipulations de corpus réalisées, commençons par préciser la finalité du travail engagé. Notre objectif n'est ni de conduire l'étude d'un phénomène linguistique en documentant nos analyses en corpus ni de développer de 
nouveaux corpus ou outils dédiés à leurs traitements. Notre rapport au corpus est en quelque sorte indirect. En effet, le produit de son exploration doit venir enrichir un dictionnaire, Les Epithetes, contemporain des textes du corpus dans lesquels son auteur a puisé la matière utile à son élaboration. Mais ce n'est que l'analyse de ce dictionnaire enrichi des attestations qui permettra de formuler ou de valider des hypothèses concernant les sources des cooccurrences inventoriées et qui nous donnera la possibilité de mener à terme l'analyse du travail lexicographique réalisé par La Porte.

La première version enrichie des Epithetes a ainsi vocation à être un outil pour l'étude du rapport entre le corpus et le texte dictionnairique, mais les résultats de ces analyses viendront à leur tour enrichir encore Les Epithetes. La version finale sera ainsi un nouvel objet éditorial à consulter pour le texte de La Porte, pour les extraits des textes de la Pléiade qui lui seront ajoutés et pour les liens et les éléments d'analyse qui résulteront des études littéraires et métalexicographiques faites, que celles-ci soient présentées explicitement ou qu'elles permettent la mise en œuvre d'affichages sélectifs.

Nous présenterons ainsi ce projet éditorial en commençant par observer la place relative des cooccurrences lexicales dans les dictionnaires en situant Les Epithetes dans la production lexicographique. Ceci nous conduira à observer que si, de nos jours, les combinaisons de mots sont souvent plus valorisées dans les dictionnaires bilingues que dans les monolingues, elles étaient plus généralement présentes autrefois et que les épithétaires constituent une classe d'objets bien identifiée mais inscrite dans la production lexicographique de son époque.

\subsection{Confrontation des articles des Epithetes et d'une sélection de dictionnaires généraux}

Les épithétaires sont des dictionnaires de cooccurrences lexicales. Des dictionnaires, ils ont le principe de traitement sous la forme de répertoires: des entrées nominales en constituent la nomenclature et des blocs de textes sont associés à ces substantifs en adresse. Ils inventorient des qualifications exemplaires (adjectifs, expressions qualifiantes ou périphrases) pour chacun des items à la nomenclature. Cette exemplarité peut être de deux ordres : soit elle se manifeste par l'usage récurrent qui en est fait chez les auteurs considérés, lorsque leurs syntagmes sont reconnus par le lexicographe comme ayant vocation à fonder une nouvelle topique, soit elle est liée à la qualité d'un nombre d'attestations plus réduit, voire unique, que le lexicographe décide de consacrer. Mais dans tous les cas, le travail du lexicographe porte sur la pertinence de collocations, selon des modalités qu'il nous faut ici resituer dans la production lexicographique actuelle et contemporaine.

En tant que dictionnaires de cooccurrences lexicales, les épithétaires peuvent être rapprochés des dictionnaires actuels consacrés aux combinaisons de mots, comme ceux qui ont été produits ces dernières années pour le français, en France ou au Québec: Beauchesne (2001) ${ }^{5}$, Zinglé et Brobeck-Zinglé $(2003)^{6}$, Le Fur (dir.) (2007) 7 et Charest, Fontaine et Saint-Germain (2012) ${ }^{8}$. Si la structure de leurs articles peut être de complexité variable, ces dictionnaires récents ont, comme celui de La Porte, des entrées nominales pour lesquelles ils aident leurs utilisateurs à mobiliser des cooccurrents idiomatiques. Mais, à la différence des Epithetes, ils ne sélectionnent pas les cooccurrences attestées dans un corpus littéraire défini et leur projet éditorial n'a pas été conçu dans un contexte linguistique comparable (cf. infra, dans cette section). 
Les épithétaires, comme ces dictionnaires de combinaisons de mots, sont des dictionnaires spécialisés. Par leur projet éditorial, et donc par la teneur de leurs articles, ils se différencient des dictionnaires monolingues et bilingues généraux que nous connaissons aujourd'hui. En effet, pour nous, les dictionnaires monolingues ont vocation à décrire le fonctionnement, dans un système linguistique donné (par exemple le français), des unités de cette langue placées en adresse d'article. Ils exploitent pour cela des définitions et une petite sélection d'exemples d'usage (cités ou forgés, montrant des contextes d'emploi typiques, incluant souvent des cooccurrents réguliers, lexicaux ou syntagmatiques). Ces composants centraux peuvent notamment être associés à des renvois synonymiques et antonymiques, voire analogiques, à des marques d'usage (diachroniques, diatopiques, diaphasiques, etc.) et au traitement d'unités polylexicales de nature variée contenant l'item en adresse d'article. Cette description est le plus souvent synchronique et peut être complétée par de brèves informations historiques (étymologie et datation de première attestation de l'item en adresse). Dans ces dictionnaires, la place de la cooccurrence est donc réduite.

Elle est un peu supérieure dans les dictionnaires bilingues, qui ont, eux, vocation à mettre en relation l'unité linguistique en adresse d'article (en langue source, le français ou le latin par exemple) avec un ou plusieurs équivalents traductionnels (en langue cible), en indiquant les conditions d'équivalence des unités des deux langues considérées. Cela motive, d'une part, l'emploi de sélecteurs de sens de l'item en langue source, s'il est polysémique, afin d'introduire les équivalents pertinents pour les valeurs sélectionnées de cet item, et, d'autre part, l'usage éventuel de marques d'usage, afin de caractériser respectivement, dans chacune des langues, les emplois des unités posées comme étant équivalentes sémantiquement. Ces équivalences traductionnelles sont le plus souvent illustrées par quelques exemples d'usage, et elles sont régulièrement accompagnées, aussi, de combinaisons de mots typiques, dont l'item en adresse est un constituant majeur, suivies chacune de leur(s) équivalent(s) traductionnel(s).

Dans ces dictionnaires généraux actuels, les types d'informations fournis sont donc diversifiés et constituent un programme d'information qui prend en considération différents aspects du fonctionnement linguistique synchronique des unités traitées; la valorisation des cooccurrences lexicales sélectionnées par les lexicographes qui les ont jugées remarquables du fait de la compositionnalité partielle seulement de leur sens, de leur typicité, de leur haute fréquence, etc., y est variable. Elles sont souvent incluses dans les exemples d'emploi sans matérialisation particulière dans les dictionnaires monolingues, mais plus visibles dans les bilingues, où elles sont plutôt sorties dans des subdivisions dédiées.

Le programme d'information des Epithetes, lui, est spécialisé dans l'inventaire de qualifications exemplaires: il a donc vocation à inventorier les cooccurrences qui le méritent. Ce dictionnaire est naturellement moins riche d'informations différentes que les dictionnaires généraux actuels, ce que peut confirmer une analyse succincte du texte de deux articles : si l'article « Amour ", cf. figure 1, comporte une liste d'épithètes puis un commentaire incluant, chose assez rare, une citation de Ronsard, l'article « Amoureus ou Aimant », cf. figure 3 (infra, §1.2.), par exemple, limite son traitement à l'inventaire initial. 
"Amour. Aveugle, cruel, impitoiable, traistre, enfiellé, jeune, fils de Venus, indiscret, folastre ou fol, bandé, guerrier, mondain, cyprien, pervers, enfant du ciel, emplumé, brutal, charnel, vitieus, captif ou captivant, pere de volupté, cauteleus, monarque, premier-né des dieux, inevitable, deceptif, tyran de la vie, meurtrier, trompeur, inhumain, felon, oiseau vainqueur, lasche, fier, moteur de l'univers, boute-feu, malheureus, enchanteur, leger, rusé, fils de la mer, archer ou archerot, soucieus, flateur, meschant, pere des muses, indomtable, maupiteus, delicat, gouverneur du monde, foible-fort, emmiellé, paphien, dieu volant, lascif, abuseur, idalien, porte-carquois, ailé, lubrique, envieus, jalous, volage, superbe, violent, joieus, amadoueur, venimeus, doux-amer, intraitable, ardent, deshonneste, insensé, opiniastre, cytherien, parjure, impatient, malin, effeminé, tendrelet, miserable, fauce-foi, rigoureus, inconstant, mensonger, dissolu, enfant de Cytheree, imperieus, rebelle, injuste, dissimulé, furieus, ingrat, puissant, fils de nature, gratieus, implacable, dionien, maistre des dieux, infame, sorcier, pipeur, doux ou doucet, enfançon, voluptueus, gouverneur des villes, inexorable, sourd, divin, aveugle, fils d'un tigre, malitieus, impudent, convoiteus, pere $d u$ souci, englueus, voletant, ambitieus, fils d'Erycine, incestueus, triumphant, audacieus, venerien, homicide, vagabond, chatoüilleus, inflexible, mauvais.

Bref veux-tu sçavoir que c'est qu'Amour?

Amour est un gouffre de maux:

Amour affole le plus sage:

Amour n'est sinon qu'une rage:

Amour aveugle les raisons:

Amour renverse les maisons:

Amour honnit la renommee:

Amour n'est rien qu'une fumee.

Quand Amour signifie Amitié, il est prins en bonne \& mauvaise part, \& est masc. \& fem. »

Est-il possible d'observer les mêmes types de différences entre le texte de La Porte et les dictionnaires généraux dont la langue objet est le français du XvI $\mathrm{I}^{\mathrm{e}}$ siècle ? Nous allons voir que ce n'est pas exactement vrai. En effet les dictionnaires généraux de français de cette époque, jusqu'à l'évolution décisive que Nicot imprime à leurs contenus en 1606, dérivent très directement de dictionnaires monolingues de latin et bilingues latin-français et fournissent, à leur instar, de longues listes de phrases ou de combinaisons de mots. Ces dernières peuvent, dans une certaine mesure, être rapprochées des inventaires de substantifs et d'épithètes de La Porte, même si les fonctions de ces contextes diffèrent partiellement: dans les dictionnaires généraux, ils ne visent pas systématiquement à montrer des emplois typiques valorisables, comme dans l'épithétaire, mais ils servent pour une bonne part à discriminer les valeurs sémantiques présentées ou impliquées dans les traductions.

La lexicographie générale actuelle est héritière des dictionnaires $\mathrm{du} \mathrm{xvI}^{\mathrm{e}}$ siècle, mais les traitements lexicographiques du français ont évolué tout comme la place de la langue française par rapport à celle du latin. Nous renvoyons à Wooldridge $\left(1977 ; 2^{e}\right.$ éd. 2010 $)$ pour une étude approfondie des "débuts de la lexicographie française» et nous nous référons à l'édition de 2010 pour les quelques éléments résumés ci-après.

Wooldridge ${ }^{10}$ rappelle que «Le Thresor de la langue françoyse de Jean Nicot [1606] est la clef du développement de la lexicographie française. Somme de quatre éditions du Dictionaire françois-latin de Robert Estienne, œuvre qui trace le chemin menant du dictionnaire latin à l'affirmation du français comme sujet premier d'un lexique français-latin, il revêt, à travers les contributions de Nicot, le caractère d'un dictionnaire français monolingue ", et « [c]e faisant, il ouvre la voie aux dictionnaires de Richelet [1680], de Furetière [1690], 
de l'Académie française [1694] et de lexicographes postérieurs [...] $»^{11}$. Ainsi le premier dictionnaire reconnu comme traitant du français en français, le Thresor de la langue françoyse, n'a été offert aux lecteurs que trente-cinq ans après que La Porte a publié Les Epithetes. Antérieurement, les éditions du Dictionaire françois-latin avaient bien le français comme langue objet, mais la métalangue, la langue de traitement, était le latin. En reprenant à Wooldridge la filiation des dictionnaires issus de ceux de Robert Estienne jusqu'à celui de $\mathrm{Nicot}^{12}$, nous observons que si la seconde édition du Dictionaire françoislatin d'Estienne (1549) comporte déjà quelques définitions dérivationnelles, « il faut attendre que Nicot prenne l'affaire en main pour voir les premières véritables analyses de sens $»^{13}$.

Afin de bien situer l'épithétaire de La Porte par rapport à ces dictionnaires généraux, intéressons-nous à celui qui en est le plus contemporain, l'édition du Dictionaire françoislatin de Jean Thierry (1564), et comparons son article « Amour » à celui du Thresor de la langue françoyse (1606), cf. figure $2 .{ }^{14}$ Pour matérialiser les différences, nous datons chaque ligne des textes, et, pour ce qui est spécifique à un texte, nous soulignons le contenu de 1564 et nous mettons en gras celui de 1606.

Figure 2 : Confrontation des articles « Amour » du Dictionaire francoislatin de 1564 et du Thresor de la langue françoyse de 1606

"Amour, Amor, Amabilitas, Charitas [1564] / Amour, m. acut. Est l'affection passionnée qu'on porte à quelque femme, si qu'il signifie chose de plus vehemente impression d'esprit que ce mot Amitié le plus souvent. Selon ce on dit, Il fait l'amour à une telle, Illam cupide amat, Il se prend aussi pour amitié qui n'a telle passion, Selon ce on dit, Il est de bonne amour, Recte amat, Amantis ingenij vir est. Et pour l'amour que le donateur porte au donataire. Quod qui donat, donatario bene cupiat, Il se prend aussi en guise de causale preposition, comme quand on dit, Je le feray pour l'amour de vous, Faciam tua (vel tui) causa, Si l'on ne veut expliquer ceste façon de parler par tels mots, A cause de l'amour que je vous porte, In gratiam amoris quo te prosequor, Amor, duquel mot Latin se fait aussi l'Espagnol, Amor, et l'Italien, Amore. [1606]

Amour ardent, Amor acer, Ardens amor. [1564 \& 1606]

Amour des-ordonné et impatient, Amor foemineus, Effraenus, Impotens, Insanus. [1564 \& 1606]

Amour evident et clair, Non obscura beneuolentia. [1564 \& 1606]

Un amour que nature a semé ou planté en nous, Insitus menti amor cognitionis. [1564 \& 1606]

Amour qui dure long temps, Longus amor. [1564 \& 1606]

Amour qui dure tousjours, Perpetuus amor. [1564 \& 1606]

[...]

Amour entre les hommes, Charitas [1564] / Mutuus inter mortales amor. [1606]

II L'amour que j'ay en toy, Tui charitas. [1564 \& 1606]

L'amour qu'il a envers luy y fait beaucoup, Permagnum pondus affert beneuolentia eius erga illum. [1564 \& 1606]

II Qui n'est point addonné à folles amours, Auersus a venereis amoribus. [1564 \& 1606]

Se deporter de ses amours, Missum facere amorem. [1564 \& 1606]

Estre en amour, se dit des oyseaux quand ils sont en chaleur et desirent s'apparier pour faire des petis. Incalescere ad coitum, re venerea agitari, appetere coitum, venerea cupiditate commoueri. Ainsi on dit les oyseaux estre en amour et s'apparier pour faire generation, Venere vrgeri ad procreandum. [1606]

Engendrer un amour, Ingenerare amorem. [1564 \& 1606]

Entrer en amour, In amorem incidere. [1564 \& 1606]

[...]

Brusler d'amours, Amore flagrare, Conflagrare amoris flamma. [1564 \& 1606] 
Faire quelque chose d'amour, qu'on a de voir quelque femme, Desiderio mulieris aliquid facere. [1564 \& 1606]

Il est hors de son bon sens, d'amour qu'il a en ceste estrangiere, Demens ex peregrina hac. [1564 \& 1606]

D'amour qu'il a en elle il n'est pas en son bon sens, Sanus non est ex amore. [1564 \& 1606] De grand amour, Ab singulari amore. [1564 \& 1606]

II Pour l'amour de moy, Causa mea, Meo nomine. [1564 \& 1606]

Il m'en fait mal pour l'amour de luy, Inuitus dico hominis causa, B. ex Liuio. [1564 \& 1606]

Pour l'amour de Dieu, Per deos immortales, B. ex Cic. [1564 \& 1606]

Envoye cestuy pour l'amour de moy, Mitte hunc mea gratia. [1564 \& 1606]

Je l'ay veu volontiers pour l'amour de luy-mesme, Illum per se vidi libentissime. [1564 \&

1606]

II Par amour, Amabo, Cum bona gratia aliquid componere. [1564 \& 1606]

II Attrayant les autres à son amour, Amabilis. [1564 \& 1606]

Chose contraignant à amour, Amatorium. [1564 \& 1606]

Portant signification de grand amour, Peramanter. [1564 \& 1606]

Un amoureux, Amans, Amator, Amasius. [1564 \& 1606]

[...]

Amoureux de muses, Philomusus. [1564 \& 1606]

$[. .]$.

Dans ces articles, il apparaît que le principe d'adressage diffère: si en 1564 ce sont conjointement Amour, Amor, Amabilitas et Charitas qui figurent en début d'article, Amour est proposé comme seule entrée lexicale en 1606, mais l'item est alors suivi d'indications de catégorie (le genre masculin étant propre aux substantifs) et d'accentuation (acut. pour aigu donc accentué) ${ }^{15}$, suivies elles-mêmes d'un développement explicatif en français, auquel sont mêlées quelques expressions latines, et une indication étymologique qui explique que du même étymon latin Amor sont issus l'espagnol Amor et l'italien Amore. Par ailleurs, l'équivalent latin donné pour Amour entre les hommes diffère : c'est l'un des items en adresse, Charitas, en 1564 et l'expression Mutuus inter mortales amor en 1606. Une dernière différence consiste dans le fait que le texte de 1606 traite Estre en amour avec, en français, une explication décrivant cet état et le présentant comme spécifique aux oiseaux ("se dit des oyseaux quand [...]»), suivie d'une séquence d'expressions latines exprimant des états comparables - mais qui ne sont peut-être pas propres aux oiseaux -, puis d'une information sur l'expression en français de cet état propre aux oiseaux («Ainsi on dit les oyseaux estre en amour [...] ») et de la traduction latine de cette dernière.

Pour le reste, les deux textes sont identiques, avec, sur chaque ligne, des combinaisons de mots en français ${ }^{16}$ qui figurent en première position et en italique (comme tout ce qui est en français), séparées par une virgule de leur(s) équivalent(s) latin(s) écrit(s) en romain (3 pour Un amoureux). Bien qu'il soit difficile d'apprécier le degré de lexicalisation de ces énoncés en français, il semble en tout cas que certains puissent être des combinaisons de mots d'une certaine idiomaticité (comme Amour ardent ou Brusler d'amours) alors que d'autres (par exemple Un amour que nature a semé ou planté en nous, Faire quelque chose d'amour, qu'on a de voir quelque femme ou Amoureux de muses) pourraient n'avoir été sélectionnées que parce que dans le Dictionarium latinogallicum elles servaient de métalangue explicative du latin et que lors du "retournement de nomenclature" de celuici pour créer le Dictionaire francoislatin ${ }^{17}$ elles se sont retrouvées en première position.

De même, dans les éditions de 1564 et de 1606, ce sont les mêmes alinéas (L'amour que j'ay en toy; Qui n'est point addonné à folles amours; Miserable d'amour; Pour l'amour de moy; Par amour; Attrayant les autres à son amour) qui débutent par le symbole «II ", l'unique 
codification structurelle symbolique employée et qui constitue un séparateur de sousensembles sémantiques.

Si c'est effectivement dans le Thresor de la langue françoyse de 1606 qu'apparaissent les composants que nous retrouvons dans les dictionnaires généraux monolingues et bilingues actuels ${ }^{18}$, il est patent que la distance entre le texte de La Porte, en tant que dictionnaire spécialisé, et le Dictionaire francoislatin de 1564 est moindre que celle qu'il entretient avec les dictionnaires généraux d'aujourd'hui. En effet, nous trouvons, dans le dictionnaire de Thierry, un important inventaire de combinaisons de mots dont le statut est malaisé à déterminer, mais qui (outre les énoncés explicatifs) semblent pouvoir aller de celui de cooccurrences remarquables à celui de simples contextes d'emploi typiques, et dont la présence paraît être motivée, comme dans le dictionnaire de La Porte, par le souhait de soutenir l'expression en langue française de ceux qui consultaient les articles. Toutefois, et il s'agit là d'une différence essentielle, La Porte ne recourt pas au latin comme métalangue, les commentaires qu'il propose sont rédigés en français, comme dans le Thresor de la langue françoyse de 1606, et ne sont pas traduits. Il s'agit en effet pour lui de revendiquer pour la langue française un corpus épithétique capable de rivaliser avec ceux que la lexicographie en langue latine mettait en valeur, pour le néo-latin, depuis le début $\mathrm{du} \mathrm{XVI}^{\mathrm{e}}$ siècle.

\subsection{Les Epithetes parmi les dictionnaires de cooccurrences du $x \mathrm{Il}^{\mathrm{e}}$ siècle}

La spécificité des Epithetes de La Porte au sein des dictionnaires contemporains du même type doit donc également être resituée. En effet, par ce recueil, La Porte s'inscrit dans une tradition déjà bien attestée en langue latine, mais il innove en la transposant dans la langue française. S'il existe quelques lexiques de ce genre au Moyen Age, généralement scolaires ${ }^{19}$, l'impulsion est vraiment donnée au $\mathrm{XvI}^{\mathrm{e}}$ siècle par Jean Tixier de Ravisy (Ravisius Textor), auteur d'un Specimen Epithetorum (1518) puis d'un recueil d'Epitheta (1524) qui recensent des épithètes poétiques latines avec leurs références, des indications prosodiques et des commentaires ${ }^{20}$. L'essor de tels ouvrages s'explique par la philologie humaniste ainsi que par le contexte de l'encyclopédisme, et il nous faut, pour bien les comprendre, prendre ici nos distances à l'égard de la perspective lexicographique traditionnelle qui voit dans ces dictionnaires dits "partiels» des précurseurs de l'évolution des dictionnaires monolingues telle qu'elle vient d'être évoquée ${ }^{21}$. En effet, si la recherche de cooccurrences de substantifs et d'épithètes peut certes apparaître comme un moyen d'affiner le sens à une époque où la structuration sémantique des dictionnaires de mots et l'exploitation des relations sémantiques en leur sein sont encore exploratoires, elle correspond tout autant à des pratiques scolaires - notamment, en latin, celle de la versification -, à des conceptions rhétoriques dominées par le souci de l'amplification, à des débats poétiques sur les grands modèles dignes d'être imités - comme Homère et Virgile, autour de la question de l'épithète homérique ${ }^{22}$ - et à un public d'utilisateurs essentiellement tourné vers la composition poétique. Il correspond aussi sans doute à un rapport au monde, moins essentialiste qu'exploratoire, avant le retour en force du concept et, par là même, du substantif au XVII ${ }^{\mathrm{e}}$ siècle ${ }^{23}$. Les pratiques poétiques du $\mathrm{XVI}^{\mathrm{e}}$ siècle montrent que cet intérêt pour l'épithète s'ancre dans les conceptions épistémologiques de cette époque. Il correspond ainsi moins à un projet lexicographique 
«partiel» qu'à une façon globale d'envisager les mots et les choses, par un biais spécifique.

Régent du collège de Navarre, Textor s'adresse à un public scolaire, mais aussi aux poètes. Admirateur d'Erasme, qui avait théorisé l'idéal esthétique de l'abondance (la copia), comprise comme une réserve de ressources stylistiques exploitables ${ }^{24}$, et qui avait luimême recensé des milliers de proverbes dans ses Adages $^{25}$, il offre aux poètes et poétaillons une ample réserve d'épithètes, célèbres ou rares et innovantes, et propose des modèles à imiter. Son ouvrage, maintes fois réédité, a durablement influencé la production poétique du $\mathrm{XVI}^{\mathrm{e}}$ siècle avant de se spécialiser dans un usage scolaire au XVII siècle, et d'être englobé dans une série d'ouvrages scolaires généraux compilant l'ensemble des ressources utiles à la versification ${ }^{26}$. D'autres épithétaires, cicéroniens, virgiliens, grecs, se développent à cette époque, dans le contexte du débat sur les modèles - plus ou moins centrés sur un auteur et puristes, ou au contraire ouverts sur une imitation plurielle - et de la redécouverte de la langue grecque.

La Porte transpose cette entreprise en langue vernaculaire. Issu d'une famille d'imprimeurs intimement liée à la Pléiade ${ }^{27}$, il veut, comme elle, « défendre et illustrer la langue française " à l'égard du latin ${ }^{28}$. Il consacre aussi le succès de Ronsard, le " prince des poètes ", qui est sa source principale. La versification en langue française ne faisant pas (ou très peu) partie des exercices scolaires à l'époque, l'ouvrage s'adresse aux poètes, apprentis ou confirmés. Il aura lui aussi un grand succès jusqu'au XVII ${ }^{\mathrm{e}}$ siècle et sera même traduit en néerlandais ${ }^{29}$.

Antérieur à la grammaticalisation survenue au XVII siècle, l'épithétaire de La Porte témoigne d'une conception plus rhétorique que grammaticale de l'épithète ${ }^{30}$. C'est ainsi qu'il recense toutes sortes d'expansions nominales, périphrases, antonomases, adjectifs, participes, etc., quelles que soient leur nature et leur fonction dans les textes sources. À l'article «Amour », cf. figure 1 (supra, § 1.1.), par exemple, figurent, à côté des formes adjectivales, les périphrases fils de Venus, enfant du ciel, père de volupté, etc. ; des substantifs à valeur d'antonomases comme archer ou archerot; et, parmi les adjectifs proprement dits, de nombreux néologismes caractéristiques de la Pléiade ${ }^{31}$, notamment des épithètes mythologiques calquées du grec (cyprien, paphien, idalien, etc.), des dérivations imitées de Pétrarque (enfiellé, emplumé, englueus, etc.) et des adjectifs composés (boute-feu, foible-fort, porte-carquois, doux-amer, etc.). La citation de Ronsard présente dans le commentaire du relevé32 ouvre encore ces possibilités par des métaphores (gouffre de maux, rage, fumée) et diverses actions verbales propres à susciter d'autres épithètes éventuelles : pourquoi pas renverse-maisons par exemple? Plus fantaisiste que les Epitheta de Textor, l'épithétaire de La Porte présente ainsi des appariements stimulants de substantifs et d'épithètes. Il entérine des innovations de la Pléiade, en écarte d'autres, en invente, en suggère, et s'en amuse.

Voyons donc quelques exemples de cette désinvolture féconde sans quitter le terrain amoureux. La confrontation, en figure 3, de l'article "Amoureus ou Aimant» (sans commentaire, cf. § 1.1.) et de son équivalent féminin "Amoureuse ou Amante " (constitué de quatre listes d'épithètes séparées par trois commentaires, qui conditionnent chacun le contenu de la sous-liste qui le suit) présente deux cas extrêmes sur une même page : le commentaire métapoétique qui organise les qualifications de la bien-aimée opère un classement axiologique, selon les situations d'énonciation, sous un propos moralisateur et quelque peu misogyne; il procède aussi à une analyse stylistique - qui prend position au sein des débats contemporains sur le pétrarquisme ${ }^{33}$ - et lexicologique 
- entérinant les adjectifs composés à valeur d'oxymores (douce-contraire, douce-ennemie, etc.), qui sont un des marqueurs du style de la jeune Pléiade.

Figure 3 : Articles « Amoureus ou Aimant » et «Amoureuse ou Amante » des Epithetes

"Amoureus ou Aimant. Passionné, transi, douloureus ou doulouré, secret, dispos, gambadeur, frisque, mignon ou mignard, vanteur, glorieus, miserable, esmerillonné, fol ou folastre, brusque, plaisant, aveugle ou aveuglé, insensible, muet, pensif, desloial, triste, petulant, soldat de Cupidon, bigarré, abesti, fretillant, fantastique, menteur, brave, luxurieus, muguet, parfumé, decouppé, gorgias, fringuant, marmiteus, resveur, jeune, morne, affable, miste, gentil, propre, beau danseur, lascif, gaillard, trompeur, mondain, langoureus ou alangouré, parjure, courtois, douillet, frisé, soupçonneus, dameret, esventé, baladin, freluquet, accort, impatient, volage, double, prodigue de gambades, couart, incertain, gouteus, sot, peigné, dorloté, fraizé, ministre d'amour, esclave, enrheté, beant, doux-parleur, bragard ou bragueur, poursuivant, esveillé, popin, chetif, solitaire, donneur d'aubades, loup-garou, maniacle, enragé, impudique, empoinct, asservi, gratieus, endemené. Amoureuse ou Amante. Belle, rusee, lascive, variable, sote, desdaigneuse, sadinette, folastre, jolie, gaillarde, courtisane, piperesse, joïeuse, affettee, gentille, plaisante, jalouse, mignonne, propre, gente, frisque, godinette, blanche, polie, vermeille, paree, miste, popine, gorriere, succree, pompeuse, dissolue, atouree, cointe, brave, fardee, courtoise, pimpante, attifee, jeune, mignote, infidele, musquee.

Ce sont à mon jugement les mieux convenables epithetes qu'on puisse donner à ces foles amoureuses, jaçoit que de la plusgrande partie noz amoureus transis n'aient accoustumé d'user en leurs escrits, principalement vers celles dont ils esperent obtenir quelque faveur, lesquelles ils appellent

Deesses, angelettes, divines, celestes, sainctes, sacrees, roines, princesses, dames, maistresses, compagnes des dieux, chef-d'œuvres de nature, douces jolieres, petites colombelles, tourtres, leur tout, bien, heur, vie, ame ou amelette, cueur, œil ou œillet, bouche, sang, amour, repos, santé, espoir ou esperance, douceur, plaisir, attente, souhait, connoissance, accueil, object, esmoi, liesse, esprit, appui, soulas, esbat, suffisance, but, ris, desir, pensee, souci, bonté souveraine, entelechie, i. perfection, soleil, lumiere, clarté, muse, charite, delices, moictié, foi, promesse, chaleur, flamme, diamant, perle ou perlette, miel, succre, printemps, fleur, rose, lis, basme, violette :

Et quand ils veullent exprimer les contraires passions d'amour, ils usent de ces mots,

Douce-contraire, douce-ennemie, douce-guerriere, douce-amere, douce-fiere, douce-rebelle, douce-inhumaine, aigre-douce, belle-rebelle, humble-fiere :

Mais si madame l'Amoureuse connoissant possible le maigre proffit qu'on peut tirer de telles personnes, de qui la monnoie est ordinairement en papier, fait la sourde oreille à leurs requestes, elle sera lors nommee,

Ingrate, cruelle, obstinee, audacieuse, dure, indocile, outrecuidee, ennemie, sote, glacee, inconstante, esprit de roche, ame faite de fer, rebelle, inexorable, guerriere, farouche, rude, froide, pillarde, jouet à vent, orgueilleuse, volage, inhumaine, opiniastre, morne, lente, meurdriere, impiteuse ou impitoiable, mauvaise, facheuse. "

Il est aussi notable que le masculin et le féminin bénéficient de deux articles séparés, que les entrées de ces articles apparient les formes synonymiques « Amoureus ou Aimant » et "Amoureuse ou Amante", alors que sur la même page, cf. figure 4, l'homonymie du substantif «Amourettes» occasionne deux entrées distinctes, l'une pour le diminutif d'« Amour ", lui-même traité à part, et l'autre pour la fleur qui porte ce nom : on mesure ici l'ampleur du travail de classification sémantique effectué par La Porte.

Figure 4 : Articles « Amourettes » des Epithetes

«Amourettes. Gaies, mignardes, jolies ou joliettes, douces, folastres, jeunes, plaisantes, joïeuses, gaillardes, feminines, gentilles. 
Amourettes. Palles, fretillantes, blaffardes, tremblotantes, fleuries, inconstantes.

Nom de fleur qui est vulgaire. »

Tel quel, l'épithétaire de La Porte témoigne de l'importance poétique des expansions nominales, de leur valeur métaphorique, ainsi que d'un rapport au monde. Éditer La Porte, en identifiant ses sources et ses choix, présente ainsi un quadruple intérêt : sur le plan lexicologique, il s'agit de prendre la mesure des apports de la Pléiade, et des critères du lexicographe négociant avec un usage qui n'est pas toujours prêt à les recevoir ; sur le plan rhétorique, d'analyser l'élaboration des tropes dans les textes; sur le plan épistémologique, d'envisager un rapport particulier entre les mots et les choses; et en termes de réception, enfin, nous n'excluons pas de pouvoir exploiter un jour cet outil dans d'autres corpus témoignant de l'influence de la Pléiade ou de l'épithétaire lui-même 34 .

\section{Manipuler des corpus du $X \mathrm{~V} \mathrm{I}^{\mathrm{e}}$ siècle afin d'élaborer une édition électronique enrichie des Epithetes}

Attachons-nous maintenant à présenter le dictionnaire électronique en cours d'élaboration, dans sa première version enrichie, celle destinée à documenter les analyses littéraires et métalexicographiques, comme dans la version poussée à son stade ultime d'enrichissement et destinée à être publiée en version électronique. Dans ce but, nous présenterons la structure XML prévue pour l'épithétaire enrichi (son modèle de document, cf. § 2.1.), avant d'évoquer les contraintes spécifiques aux traitements de corpus du $\mathrm{XVI}^{\mathrm{e}}$ siècle et aux compétences stylistiques que requièrent les analyses à mener dans le cadre de notre projet (\$ 2.2.), puis de nous concentrer sur Les Amours de Ronsard pour présenter quelques problèmes manipulatoires archétypaux (\$ 2.3.).

\section{1. Élaborer une version enrichie et balisée en XML de l'épithétaire de La Porte}

À partir du texte original imprimé des Epithetes, une succession de tâches s'imposait pour la réalisation d'une édition électronique enrichie de cet épithétaire. Nous ne reviendrons pas sur chacune ici, mais il semble opportun de présenter le processus dans son ensemble avant d'exposer le modèle de document XML du texte dictionnairique enrichi.

Tout devait commencer par la saisie du texte du dictionnaire, qui n'est pas encore disponible dans une version électronique exploitable ${ }^{35}$ et dont le coût de numérisation avec reconnaissance optique des caractères serait dissuasif compte tenu des spécificités de ce texte comme de tous ceux publiés au XVI ${ }^{\mathrm{e}}$ siècle (cf. § 2.2.).

Ensuite, outre le balisage du texte, un important travail de standardisation des graphies a dû être effectué puisque l'orthographe n'est pas stabilisée au XVI siècle (cf. § 2.3.). Ce premier enrichissement du dictionnaire a consisté à annoter chaque substantif ou épithète afin d'associer une graphie identique à chaque occurrence de chaque item du texte original. Ces graphies standardisées permettent :

- de retrouver les occurrences des épithètes présentes dans différents articles, afin de proposer à terme un accès de consultation par celles-ci ;

- mais aussi, et surtout, de procéder aux appariements des cooccurrences relevées par La Porte avec celles qui sont présentes dans les textes de la Pléiade, afin d'intégrer les 
citations pertinentes au texte dictionnairique. La dernière manipulation implique, évidemment, que les graphies des items de ces textes soient également standardisées quand nous les traitons. ${ }^{36}$

Enfin, une fois les attestations des cooccurrences dans les textes de la Pléiade explorés reliées aux cooccurrences inventoriées par La Porte, l'épithétaire enrichi offrira une première version électronique (une version en HTML publiée à partir du texte balisé en XML) qui permettra d'affiner l'analyse des sources de La Porte en présentant tous les contextes d'attestation connus et en offrant donc un environnement de travail riche. Quand des hypothèses auront pu être formulées concernant la relation la plus probable entre une citation et une cooccurrence relevée (est-ce une source, un réemploi, etc. ?), le fruit de cette étude viendra enrichir encore le document XML et sera exploité pour offrir des modes de consultation additionnels.

Attardons-nous maintenant sur la présentation du modèle de document XML des Epithetes enrichi des contextes d'attestation. Le principe de balisage mis en œuvre pour le texte dictionnairique se conforme aux recommandations de la TEI P5 $5^{37}$ mais n'exploite pas les codifications qui y sont propres aux dictionnaires ${ }^{38}$. Nous avons en effet préféré exploiter des éléments plus communs, qui se prêteront peut-être mieux aux échanges avec des partenaires éditoriaux non spécialistes de dictionnaires, puisque le modèle de document retenu s'apparente davantage à ceux qu'ils manipulent pour d'autres types de contenus textuels.

Pour exposer le modèle de document XML et le processus d'élaboration des versions XML et HTML, nous nous concentrerons sur l'article «Bacche ou Bacchus ». Son texte original est présenté en figure 5 et un premier prototype d'affichage HTML enrichi l'est en figure 6 (il indique les auteurs des attestations des cooccurrences ainsi que, pour Ronsard, les volumes de l'édition proposée par Laumonier - « Lm »-, cf. n. 3) $)^{39}$.

Figure 5 : Article « Bacche ou Bacchus » des Epithetes

Bacche ou Bacchus. Joieus, amiable, effeminé, vineus, porte-lance, thebain, martial, lenean, cuisse-né, nysean, enfançon de Silene, recreatif, ïvrongne, pere de verité, Roi de naxe, aonien, double-corne, vigneron, œil-toreau, furieus, porte-lyerre, omadien, dieu du vin, triete, rompsouci, archete, bromien, alme, hymenean, deuis, bassare, eubolien, rustique, double, gay, hospitalier, rouge, nictolien, trigone, solitaire, vangeur, manique, nomien, germe des dieux, premier, dernier, beaucoup-forme, meonien, noble, dissolu, enfant, doux, grandime, euchien, domteur des Indes, porte-sceptre, philonien, nourri-vigne, balleur, lyssien, bonime, tendret, lyean, maistre des Satyres, ayme-pampre, lychnite, mol, potelé, mystic, baladin, lesbien, fils de semele, insensé, ogygien, cornu, belliqueus, silenien, inventeur, thyonean, jeune, eldean, fol, dieu nouricier, thracien, vieillard, pcean, esraphiot, indien, phanete, libre, valeureus, joufflu, deux-fois-né, agnien, guerrier, semelien, eshonté, hardi, triumpheur indien, sacré, genereus, dircean, roi des Pions, vieil, jouvenceau, dieu des indes, satyre, linean, prince des vins, dieu chevrier, nourrisson de Silene, vainqueur indien, empampré.

Bacchus fils de Iupiter \& de Semele a esté reputé dieu du vin, \& est prins aucunesfois pour le vin mesmes. Les poëtes feignent qu'il a esté deux fois né, \& qu'il est sorti de la cuisse de Iupiter. Il a presque voiagé par tout le monde, \& a domté plusieurs nations. Les Indiens ont esté par lui surmontez, \& a premier triumphé d'iceux. Il a aussi monstré la maniere de vendre \& d'acheter, \& dict on qu'il a inventé plusieurs choses. 
Figure 6 : Prototype d'affichage de l'article «Bacche ou Bacchus » limité à sa liste d'épithètes enrichie d'indications d'auteurs et de volumes d'attestation dans l'édition de Ronsard proposée par Laumonier

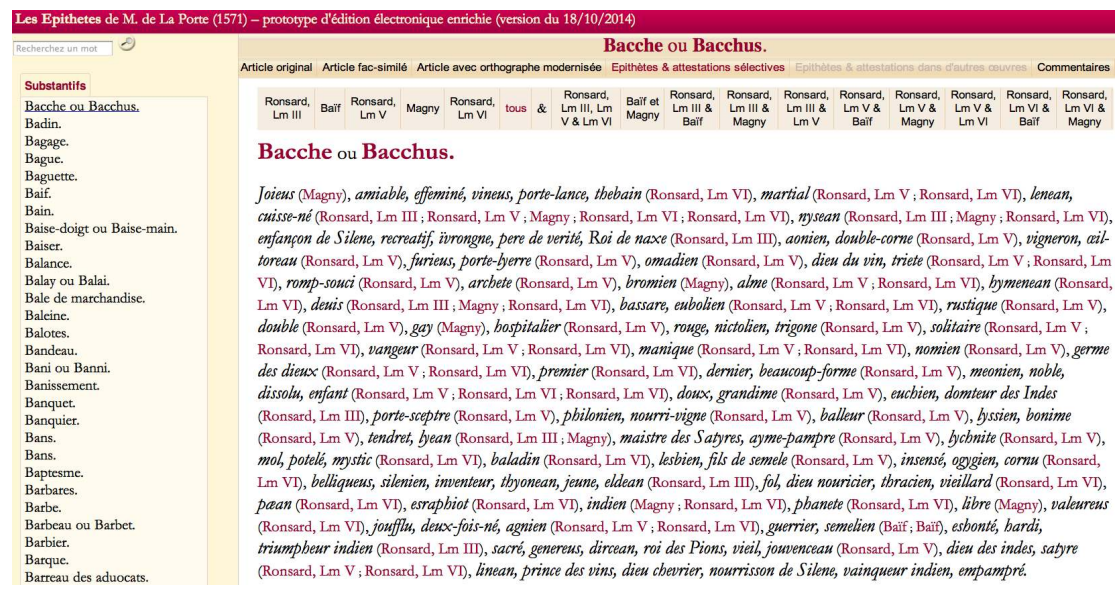

L'édition électronique, en HTML, est produite à partir d'une source XML qui fusionne le texte de La Porte, les segments des textes de la Pléiade au sein desquels les cooccurrences lexicales sont attestées et certaines indications procédant de l'analyse de ces contextes. Une visualisation du modèle de document XML des articles enrichis est présentée en figure 7, avant que ne le soit un extrait de l'article «Bacche ou Bacchus » balisé en XML (xmlisé) en figure 8.

Figure 7 : Modèle de document pour le balisage des articles en XML

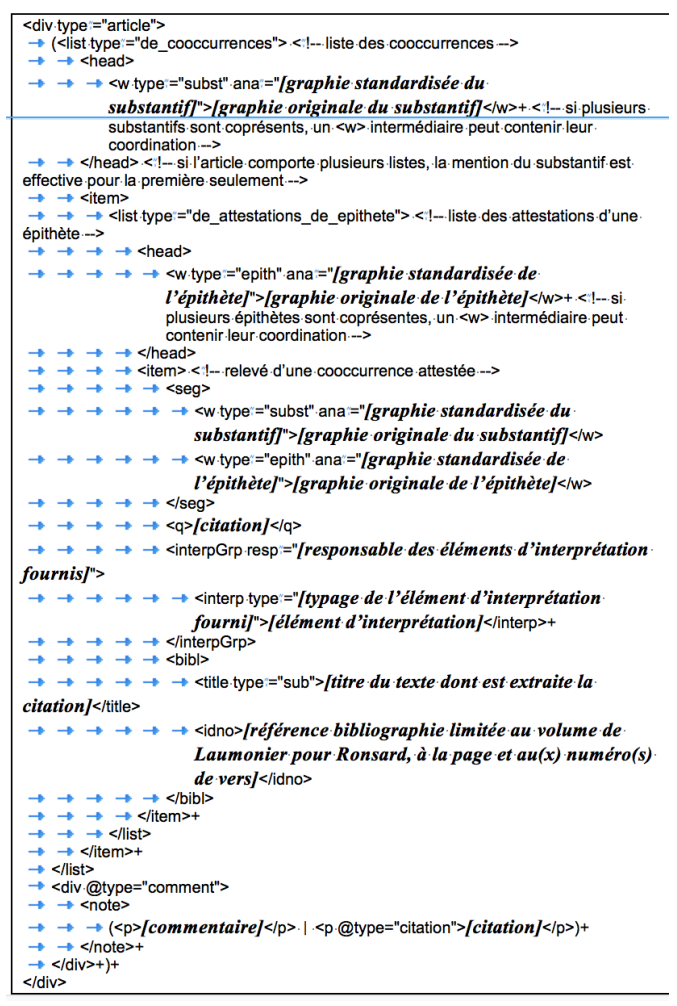

Le modèle de document présenté en figure 7 impose la structure suivante :

- Chaque article constitue un <div> porteur d'un attribut @type="article" contenant un élément <list> porteur d'un attribut @type="de_cooccurrences" (la liste des 
cooccurrences constituée par le substantif (ou l'un des substantifs) en adresse d'article et par chacune des épithètes listée ensuite). Le(s) substantif(s) en adresse constitue(nt) le $<$ head $>$ de cette liste et chaque épithète un <item>.

- Le $<$ head $>$ de la liste de cooccurrences contient un ou plusieurs éléments $\langle\mathrm{W}\rangle$, selon s'il y a un ou plusieurs items en adresse d'article (et pour leur éventuelle coordination), chaque substantif étant typé (au moyen de l'attribut @type ="subst") et associé à une annotation qui fournit sa graphie standardisée (@ana ="Bacchus" en figure 8).

- Chaque <item> de la liste de cooccurrences contient lui-même un élément <list> porteur cette fois d'un attribut @type ="de_attestations_de_epithete", dont l'épithète constitue le $<$ head> et dont les éléments <item> correspondent aux différentes citations référencées et analysées, comme celle présentée pour Bacchus double-corne en figure 8.

- Chaque <item> de la liste d'une épithète fournit le relevé d'une cooccurrence attestée. Il contient lui-même :

- un élément <seg>, qui présente les cooccurrents (pour Bacchus double-corne, le nom est absent du contexte mais restitué par l'interprétation, il figure donc entre parenthèses),

- un élément $<q>$, qui montre l'attestation relevée,

- un élément <interpGrp>, qui fournit les données d'interprétation établies sous la responsabilités d'un analyste (ce que code l'attribut @resp="APPM"40), chaque information donnant lieu à un élément enchâssé <interp> porteur d'un attribut en typant la nature, et qui informe, en figure 8, sur le fait que double-corne est interprété comme un adjectif (substantivé) en apostrophe constituant une antonomase ${ }^{41}$,

- et, enfin, un élément <bibl>, qui référence l'attestation relevée et analysée au moyen de deux sous-éléments, l'un pour le titre du texte (<title type ="sub">) et l'autre pour la localisation du segment cité (<idno>). 


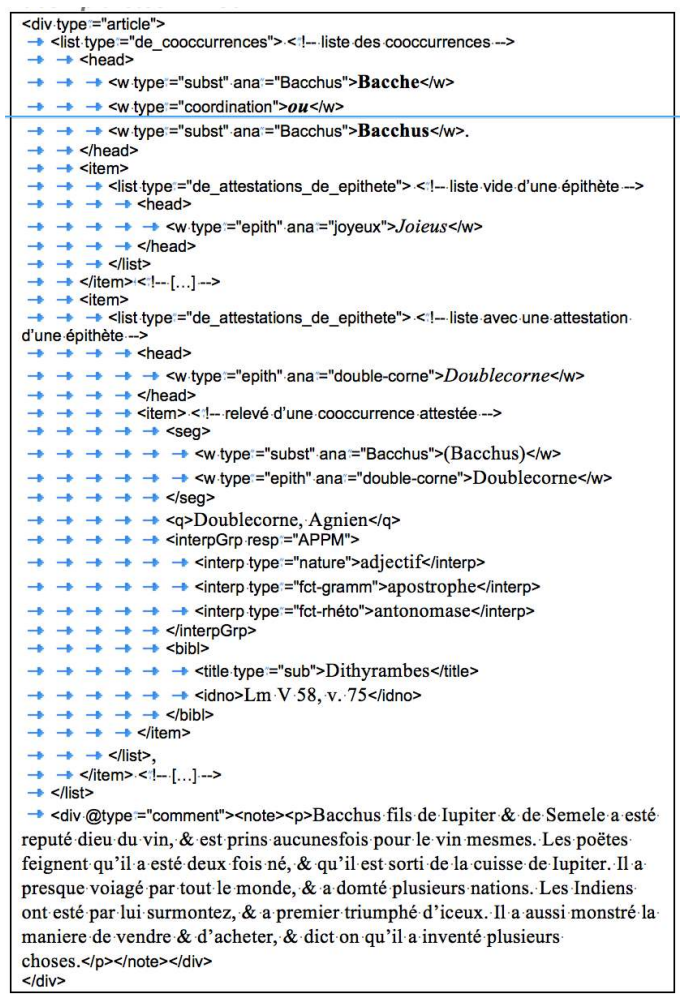

Un article comme "Amour», cf. figure 1 (cf. § 1.1.), comporte en outre une citation balisée comme un <p> porteur de l'attribut @type="citation" enchâssé dans le commentaire.

Nous avons, dans un premier temps, réservé le traitement des épithètes contenues dans les commentaires des articles ${ }^{42}$, mais nous devrons les traiter ultérieurement, cf. $§ 3.1$.

\subsection{Contraintes spécifiques des corpus du $x v l^{e}$ siècle et compétences requises pour les manipuler}

Un mot d'explication s'impose ici concernant les contraintes spécifiques qui ont déterminé les procédures que nous avons adoptées pour les manipulations des Epithetes comme du corpus de textes. Elles sont liées à la fois à l'ancienneté du corpus et à sa construction autour d'un objet rhétorique.

La nécessité de transcrire le texte de l'épithétaire et d'en standardiser l'orthographe a déjà été évoquée. Pour déroutante qu'elle puisse paraître dans le cadre de la linguistique de corpus électroniques, cette pratique de la transcription n'a rien d'extraordinaire dans le cadre de l'édition des textes du Xvi ${ }^{\mathrm{e}}$ siècle : elle est, au contraire, la norme, en l'absence de version numérique disponible et $\mathrm{du}$ fait de la faible lisibilité des caractères d'impression de cette époque, peu déchiffrables par le biais d'un scanner. ${ }^{43}$

En ce qui concerne les textes sources potentiels, la situation est différente, car il en existe parfois des versions numérisées ; mais, outre le fait que toutes ne présentent pas le même degré de fiabilité, se pose la question des différents états du texte au fil de ses rééditions, parfois très nombreuses, et du choix de l'édition de référence. L'objectif de ce projet, qui est de réaliser un produit exploitable par la communauté scientifique, implique d'indexer 
les renvois bibliographiques sur les éditions de référence des auteurs dépouillés par La Porte, en incluant leurs variantes lexicales. Pour Ronsard, par exemple, l'édition choisie est celle de Paul Laumonier cf. n. 3, considérée comme édition de référence, et qui fournit le premier état du texte ainsi que ses variantes ultérieures.

La standardisation orthographique, qui s'impose aussi bien pour La Porte que pour ses sources, afin de permettre la confrontation des cooccurrences observées, est indissociable de cette question des différents états du texte. Elle ne va pas de soi pour les lexies sorties de l'usage - pour lesquelles les entrées des dictionnaires de référence de l'Ancien et du Moyen Français peuvent nous aider -, ni pour les néologismes, très nombreux dans la poésie de la Pléiade (comme paphien, cf. $\$ 1.2$.). Se pose en outre le problème des transcriptions de variantes phonétiques minimes - voire inexistantes selon la prononciation de l'époque, même si elles paraissent plus importantes aujourd'hui, comme certaines nasalisations -, susceptibles de faire obstacle à l'identification alors qu'elles correspondent peut-être simplement à des choix de présentation du lexicographe à partir des auteurs qu'il a recensés.

Notre mode opératoire consiste à partir des sources majeures de La Porte - principalement Ronsard et plusieurs autres poètes de la Pléiade - et à procéder sur elles soit à un balisage des substantifs et des épithètes du corpus afin d'extraire leurs cooccurrences (cf. § 3.1.), soit à des relevés manuels systématiques de cooccurrences (cf. §3.2.), pour les confronter ensuite aux sélections effectuées par La Porte. Il aurait pu sembler plus logique d'adopter le processus inverse, en partant des cooccurrences retenues dans l'épithétaire pour en retrouver les attestations en effectuant des recherches de chaînes de caractères. Nous n'excluons d'ailleurs pas de procéder ainsi pour les corpus secondaires, une fois les identifications faites sur les textes majeurs; et c'est ce que nous ferions aussi pour ces derniers si nous ne recherchions que les attestations de ce qui a exactement été relevé par La Porte. Mais en procédant ainsi, nous manquerions l'essentiel de son travail interprétatif et créatif (cf. §3.2.). En d'autres termes, le type d'objets que nous recherchons dans ces textes n'y est pas toujours strictement attesté. De plus, cette exploration fine, effectuée soit par balisage soit par relevés manuels enrichis d'annotations rhétoriques, nourrit l'analyse proprement stylistique de ces corpus.

Une dernière série de contraintes à considérer touche enfin à la définition de l'épithète au XVI ${ }^{\text {e }}$ siècle. En effet, la définition rhétorique de celle-ci, comme expansion du nom non seulement adjectivale, mais potentiellement prépositionnelle ou propositionnelle, incluant la possibilité de la coréférence, de l'adjectivation des gérondifs et de figures telles que la métaphore, l'antonomase, la périphrase ou l'oxymore adjectivé, démultiplie la part d'interprétation dont dispose le lexicographe, et rend malaisée la description formelle de tout syntagme nominal qui pourrait constituer pour l'épithétaire un candidat valide. Nous touchons ici aux difficultés inhérentes à l'application d'outils électroniques à des faits stylistiques: tout l'intérêt de cette exploration tient précisément au fait que l'épithétaire de La Porte n'est pas un simple relevé de cooccurrences de substantifs et d'adjectifs, mais un dictionnaire poétique. 


\subsection{Difficultés d'exploration des textes de la Pléiade : problèmes archétypaux posés par les Amours}

Les difficultés de manipulation que posent les textes de la Pléiade peuvent être illustrées par le premier recueil des Amours de Ronsard, un ensemble de textes dont le traitement a fait progresser notre représentation des difficultés à prendre en compte ${ }^{44}$. Nous insisterons ici sur deux points, tous deux liés à la question de l'édition consultée par La Porte: l'instabilité des choix orthographiques de Ronsard et de La Porte, qui pose le problème de l'exploitation des variations attestées, et la pratique lexicographique de La Porte, qui interdit de s'en tenir à une recherche de cooccurrences strictement attestées.

\subsubsection{Standardisation orthographique et exploitation des variations attestées}

Nous avons déjà relevé que les orthographes de Ronsard et celles de La Porte pouvaient différer, ce qui a conduit à une standardisation orthographique (portée par les attributs @ana des éléments $\langle\mathrm{W}\rangle$ contenant les substantifs et épithètes dans le dictionnaire comme dans les sous-corpus ou les relevés) afin de disposer de formes automatiquement appariables. Mais la question est en fait beaucoup plus centrale, puisque l'orthographe varie d'une édition à l'autre du texte source. En effet, Ronsard a édité son premier recueil d'Amours une première fois en 1552, puis en 1553, accompagné d'un commentaire de l'érudit Marc-Antoine Muret, puis a repris en tête de la première édition de ses Euvres complètes en 1560 ce recueil rebaptisé Amours de Cassandre, symétriquement aux Amours de Marie, recueil également accompagné d'un commentaire, cette fois par Belleau. ${ }^{45} \mathrm{La}$ comparaison des variantes montre que c'est probablement à partir de l'édition commentée de 1553, et non de la toute première, que La Porte s'est intéressé à ce recueil, et qu'il y est peut-être revenu plus tard dans l'édition collective de $1560^{46}$.

Or, entre 1552 et 1553, Ronsard a procédé à une vaste révision orthographique des Amours , au cœur d'une décennie marquée par un débat orthographique entre l'ancienne école, étymologiste ou historienne (Théodore de Bèze, Guillaume des Autels), qui vise à garder trace du lien généalogique entre le latin et le français dans les graphies, et la nouvelle école, phonéticienne (Louis Meigret, Jacques Peletier du Mans), qui souhaite que l'orthographe soit le reflet de la prononciation. La position de Ronsard fluctue au fil des années ${ }^{47}$, tout comme il hésite entre la volonté exigeante et élitiste de créer un langage à part, et le souci de conquérir un public plus large que celui des doctes. C'est ainsi qu'après avoir marqué dans les Odes son intérêt pour certaines propositions réformistes, il était revenu à une orthographe plus traditionnelle (étymologisante) en 1552, avant de s'intéresser de nouveau en 1553 à la nouvelle orthographe (plus phonétique). Cela se traduit notamment par le remplacement du $Y$ par le $I^{48}$, de $P H$ par $F$, du $X$ muet final par $S$, par la suppression des doubles consonnes non prononcées et des lettres quiescentes (qui sont la simple trace graphique de l'étymologie des mots), ainsi que d'autres graphies traditionnelles.

Dans la perspective d'un traitement automatisé des cooccurrences, les difficultés liées aux variations graphiques du texte source pourraient apparaître à première vue comme un indice pouvant aider à identifier l'édition utilisée par La Porte. Mais, en réalité, les graphies adoptées par La Porte ne recoupent pas plus celles de l'édition de 1553 que celles de 1552. Ainsi, dans le sonnet liminaire, le syntagme jeunesse prompte, tel que l'a relevé La Porte (à la lettre $I:$ « Ieunesse »), reprend pour l'épithète la graphie de 1552, et non celle 
de 1553 (pronte) $^{49}$; le syntagme aiguillon amoureux de 1552, devenu eguillon amoureus en 1553, n'est pas relevé par La Porte, sans doute du fait de son caractère métaphorique, mais on trouve le substantif «Aiguillon » à la lettre $A$ tandis que l'épithète amoureus est généralement relevée, dans d'autres articles, avec la graphie nouvelle ${ }^{50}$; plus loin, l' homicide dart des deux versions du sonnet 4 est relevé à l'article "Dard ${ }^{51}$; la périphrase de Bellérophon dans le sonnet 15, aillé belliqueur selon la version de 1552, et celé belliqueur en 1553, est relevée en deux épithètes à l'article "Bellerophon », dont la première reprend la version de $1552^{52}$; tandis que les périphrases de l'ange du sonnet 30 , «Le truchement \& le herault des Dieux» en 1552, devenu heraut des Dieus en 1553, sont reprises dans le commentaire de l'épithétaire (s.v. "Ange») au pluriel, avec la graphie nouvelle heraut(s) de $1553^{53}$. Il est donc difficile d'en inférer une loi générale. C'est bien pourquoi la standardisation de l'orthographe s'impose comme interface nécessaire entre les cooccurrences du texte source et de l'épithétaire, ainsi que le recours à l'édition de référence des Euvres de Ronsard (cf. n. 3), qui suit le premier état de chaque recueil avec ses variantes et présente l'intérêt d'être immédiatement exploitable par quiconque souhaite revenir de l'épithétaire au texte source, même s'il lui faut éventuellement ensuite, de là, repartir en quête de l'édition effectivement utilisée par La Porte.

La standardisation orthographique que nous avons mise en place et qui adjoint des formes neutralisées aux formes originales nous permet ainsi d'opérer les manipulations utiles pour l'enrichissement $\mathrm{du}$ texte dictionnairique, mais les index de formes concurrentes qui peuvent être créés à partir des formes standardisées peuvent également alimenter des études de variantes graphiques. Néanmoins, la standardisation orthographique est une étape minutieuse, puisqu'une standardisation fautive dans un contexte donné conduirait soit à produire des appariements indus de cooccurrences relevées par La Porte et de citations référencées où les cooccurrents ne figureraient pas véritablement, soit à des non-appariements.

\subsubsection{Pratique interprétative et créative du lexicographe : les limites de la recherche d'attestations strictes}

Le fait que La Porte ait dépouillé les Amours à partir de l'édition de 1553, enrichie du commentaire de Muret, pose également la question de l'inclusion du paratexte et, plus encore, de la pratique interprétative et créative du lexicographe. En effet, La Porte ne s'inspire pas seulement du commentaire de Muret pour ses propres commentaires - largement recopiés ${ }^{54}$ - mais probablement aussi pour ses relevés, car il semble créer luimême certaines épithètes par dérivation à partir des termes du commentaire de Muret ${ }^{55}$ ou, d'ailleurs, de précisions qu'il lui ajoute ou du texte ronsardien lui-même. C'est le cas par exemple d'épithètes mythologiques comme Mygdonien pour le héros troyen Chorèbe, dont le commentaire de Muret au sonnet 4 des Amours, repris par La Porte, précise qu'il " fut un jeune homme fils d'un Phrygien nommé Mygdon ${ }^{56}$, ainsi que d'épithètes non pas créées, mais simplement déduites du commentaire du sonnet 45 , dans les articles "Ixion» (miserable) ou "Tantale» (phrygien) ${ }^{57}$. C'est aussi le cas, sur le plan épistémologique, des qualifications de l'arc-en-ciel nubileus et pluvieus: Muret, repris par La Porte, précisait en effet à propos du sonnet 65 qu'il était provoqué «par une reverberation du soleil contre la nuë », et La Porte semble aussi avoir repris directement l'adjectif pluvieus au sonnet ronsardien, où il qualifiait le temps ${ }^{58}$. Le lexicographe, soucieux d'enrichissement épithétique, applique ainsi, sans le dire, à son dictionnaire les préceptes de la Pléiade sur la création verbale ${ }^{59}$. 
Les articles consacrés à Castor et Pollux témoignent plus encore des libertés que La Porte prend à l'égard du commentaire. Le lexicographe semble ainsi avoir forgé l'épithète cebalien à partir de la périphrase des deux héros, «freres d'ÆEbalie ", dans le sonnet 50 . Il la recense sous les deux adresses "Castor » et « Pollux » ${ }^{60}$, sans pour autant reprendre le commentaire que Muret consacrait à cette épithète. Il applique, par ailleurs, l'épithète tyndaride à Castor, et non seulement à Pollux (conçu par Jupiter à la différence de son frère, fils de Tyndarée), ce qui contredit le commentaire de Muret. Cela peut s'expliquer par la tendance du lexicographe à redistribuer les épithètes récoltées d'un article sur l'autre, ainsi que par l'influence d'une autre invocation, adressée aux deux frères dans l' Hymne de Pollux et de Castor ${ }^{61}$ : La Porte a exploité celle-ci massivement dans les deux articles, en général de façon différenciée - en reprenant ecuier, cavalcadour et voltigeur s.v. «Castor » et escrimeur et guerrier s.v. « Pollux »-, sauf pour tyndaride; mais la précision de Muret sur la conception des deux frères était alors plus lointaine.

Ainsi, La Porte fond dans ses propres commentaires toutes les informations qu'il estime pouvoir enrichir lexicalement et sémantiquement le matériel épithétique qu'il récolte, $\mathrm{y}$ compris par l'insertion d'épithètes recueillies à partir de plusieurs pièces. Mais plus encore, il en recueille dans un même article à partir de substantifs différents, selon l'interprétation qu'il juge la plus pertinente. Le commentaire de l'article « Ange » est à cet égard exemplaire, cf. figure 9, puisqu'il réunit des périphrases invocatoires du sonnet 30 , qui, sous le nom d'«Ange", s'adresse en réalité au Songe érotique, mais aussi du sonnet 31, qui s'adresse aux «Daimons », êtres intermédiaires, selon le sens grec de ce mot, et nullement diaboliques, et qu'en vertu de cette acception La Porte préfère sans doute assimiler aux anges, ainsi que le diminutif mignard "Angelette", imité de Pétrarque dans les sonnets 139 et $159^{62}$.

\section{Figure 9 : Article « Ange » des Epithetes}

Ange. Divin, ailé, celeste ou celestiel, parfait, glorieus.

Parce que les Anges nous declarent la volonté de Dieu, ils sont appellez des poëtes Truchemens, herauts, messagers, postes, courriers des dieux. Ces mesmes poëtes (ausquels toute licence est permise) faisans de la nature angelique ainsi que de l'humaine, disent au feminin Angele, \& ont composé deux diminutifs Angelet \& Angelette.

La Porte fait ainsi feu de tout bois, si bien que son dictionnaire offre des listes d'épithètes aussi bien dans le commentaire que dans le relevé, les recueille aussi bien à partir des textes poétiques que de leur paratexte, et en crée ou en déduit aussi bien à partir de ces différents types de sources que du commentaire des articles, qui résulte lui-même d'une pratique de compilation.

Parmi les difficultés qui découlent de ces observations, la plus handicapante pour des analyses semi-automatiques est bien sûr qu'une partie des données lexicographiques rassemblées par La Porte ne correspond à aucune cooccurrence identifiable automatiquement, par appariement de formes lexicales, alors même que leur source se trouve dans l'édition du texte qu'il a utilisée - selon des relations de cooccurrence larges, dans le métatexte.

$\mathrm{Du}$ point de vue de l'exploration de textes sources réunis en corpus électronique, les questions de redistribution des épithètes induisent des risques de silence. En effet, même si une recherche des cooccurrences de substantifs et d'épithètes relevées par La Porte peut être doublée de celle des cooccurrences des parasynonymes des substantifs associés 
aux épithètes précédentes, seuls les parasynonymes inventoriés sont connus et donc exploitables, mais La Porte peut avoir été plus créatif. Par ailleurs, le fait qu'il faille assez régulièrement restituer le substantif implicitement présent dans les contextes (cf. supra, dans l'attestation relevée (élément $\langle q>$ ) s.v. «Bacche ou Bacchus», en figure 8 ou infra, §3.) motiverait une recherche parallèle des épithètes isolées, mais le dépouillement des données extraites pourrait s'avérer très fastidieux, et ne garantirait pas non plus que tous les substantifs candidats puissent être mobilisés. Les créations dérivationnelles, elles, pourraient nous conduire à imaginer des procédures d'appariement plus complexes, qui imposeraient de savoir analyser morphologiquement les unités lexicales relevées par La Porte (que cette analyse soit faite à la volée ou que les décompositions morphologiques soient enregistrées dans le balisage du texte dictionnairique) et de compléter les recherches de cooccurrents en corpus, qui sont actuellement faites sur la base de leur forme graphique standardisée, par des recherches conçues sur la base des constituants morphologiques. Cette entreprise serait d'une autre ampleur, puisque sa première étape consisterait à établir les décompositions morphologiques pertinentes pour les items à prendre en compte, ce que nous n'envisageons pas de faire dans le court terme, les questions orthographiques étant de fait prioritaires.

Le travail fin de relevé qui s'impose par là même s'avère en ce sens une solution pertinente aux différentes difficultés et contraintes que nous venons d'évoquer.

\section{Retour sur deux manipulations de corpus effectuées}

Concentrons-nous à présent sur deux manipulations de corpus effectuées pour la recherche des attestations des cooccurrences de substantifs et d'épithètes que La Porte a exploitées pour élaborer ses listes. Nous envisagerons successivement (i) une annotation des substantifs et épithètes au fil du texte, dans le but d'en extraire, en principe, toutes les cooccurrences, y compris celles qui n'ont pas été retenues par La Porte, ce que nous verrons appliqué au traitement des Hymnes cosmologiques de Ronsard (cf. § 3.1.), et (ii) un dépouillement manuel afin de constituer un relevé destiné à enrichir le texte dictionnairique nouvellement xmlisé, ce qui sera illustré par le traitement des pièces bachiques de Ronsard, Baiff et Magny (cf. § 3.2.).

\subsection{Procédure d'extraction semi-automatique des cooccurrences à partir de substantifs et d'épithètes balisés dans les textes : les Hymnes cosmologiques de Ronsard}

Dans un premier temps, nous avons expérimenté un repérage exhaustif des substantifs et des candidats épithètes au sein du texte des Hymnes cosmologiques de Ronsard (1555-1556) ${ }^{63}$, choisis en raison, d'une part, de l'importance de Ronsard pour les travaux de La Porte (cf. § 2.2.), et, d'autre part, de l'homogénéité générique ainsi que des spécificités stylistiques de ces hymnes. Ce premier corpus de travail compte 57500 motsoccurrences dont 1100 substantifs et 1600 épithètes annotés ${ }^{64}$. En procédant de manière inductive, à partir des textes sources et sans prise en compte des relevés effectivement faits par La Porte, nous voulions en quelque sorte nous replacer dans la situation du lexicographe qui dépouillait les textes à la recherche de cooccurrences pertinentes pour son épithétaire. 
Comme le français de Ronsard n'est pas celui que savent communément manipuler les outils du TAL, en particulier en ce qui concerne l'orthographe (cf. $\$ 2$.), nous n'avons pas cherché à automatiser la détection des substantifs et des divers types d'épithètes de ces Hymnes. Nous avons préféré adopter une méthode permettant d'enregistrer les repérages humains, en espérant que ce premier corpus annoté pourrait servir de corpus d'entraînement pour outiller les explorations d'autres textes. Le mode opératoire adopté, cf. figure 10, pour permettre d'extraire semi-automatiquement les possibles cooccurrents a donc consisté

1) à styler, dans un traitement de texte, les substantifs et les épithètes dans les textes des Hymnes au moyen de styles de caractères,

2) à convertir ce document stylé en un corpus structuré en $\mathrm{XML}^{65}$ et contenant les substantifs et épithètes balisés du fait de leur stylage antérieur,

3) à extraire, au moyen d'une transformations XSLT écrite à cette fin, les cooccurrents les plus proches (c'est-à-dire, pour chaque épithète, les premiers substantifs anté- et postposés, sans autre substantif intercalé), afin de bien repérer les items des salves d'épithètes mais de ne retenir autant que possible que ce qui qualifie chacun des substantifs,

4) à analyser les cooccurrences extraites, en sélectionnant ou en mentionnant le substantif effectivement qualifié par chaque épithète. ${ }^{66}$

Figure 10 : Extraction des substantifs cooccurrents des épithètes viste et dispos du vers 23 de l' Hymne du Ciel

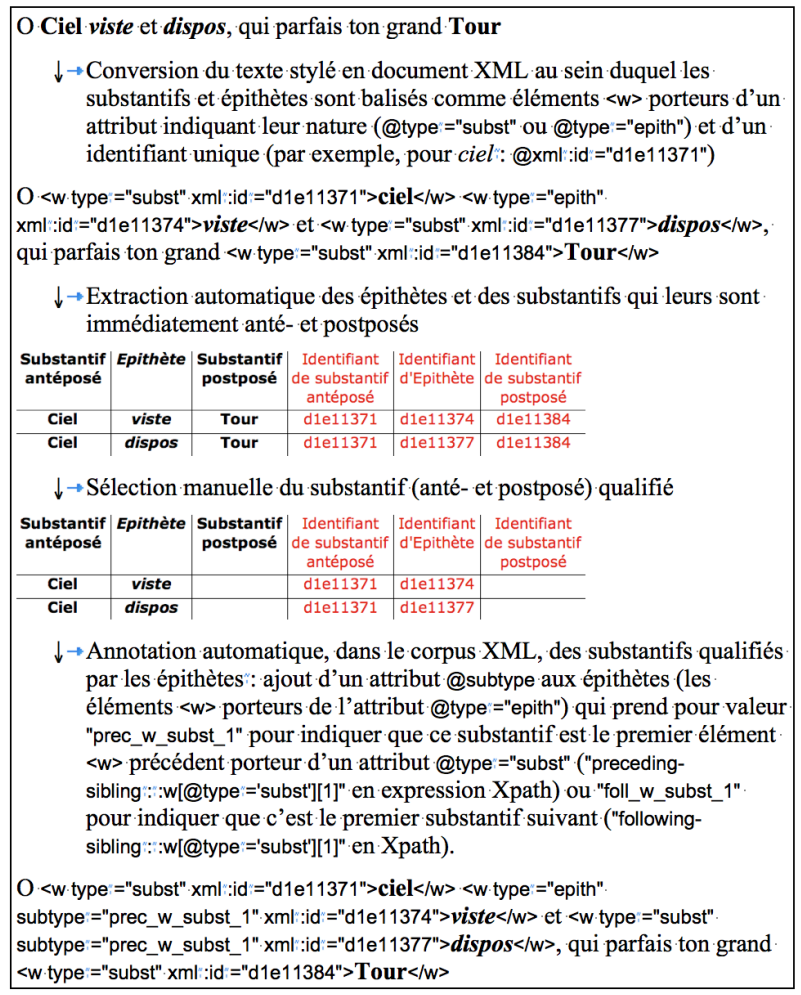

Ces manipulations ont buté sur certaines difficultés. Celles-ci tenaient bien sûr avant tout au nombre particulièrement important de cooccurrences attestées et annotées dans ces textes (plus de $1600^{67}$, puisque les épithètes ont été analysées comme qualifiant un substantif, sauf dans une dizaine de cas où elles peuvent en qualifier deux sans qu'un 
choix soit faisable), ce qui a produit une lourde quantité de données à étudier. Elles tenaient aussi aux spécificités poétiques des Hymnes, qui présentent un grand nombre d'invocations litaniques, sans que le substantif du référent célébré soit toujours exprimé. C'est le cas par exemple au terme de l'Hymne de l'Éternité en figure 11.

Figure 11 : Hymne de l'Éternité, p. 254, v. 135-137

Je te salu' Deesse au grand œil tout-voyant, (135)

Mere du grand Olympe au grand tour flamboyant,

Grande mere des Dieux, grande Royne et Princesse

Ici l'invocation adopte un tour antonomasique et périphrastique reposant sur des substantifs communs, Deesse, Mere, Royne et Princesse, expansés par l'adjectif épithète grand qui a pour fonction rhétorique de construire le trope d'excellence ${ }^{68}$, et par des compléments du nom qui font eux-mêmes l'objet d'expansions adjectivales et prépositionnelles récursives, générant des suites de groupes prépositionnels emboîtés ( $a u$ grand ceil tout-voyant, du grand Olympe au grand tour flamboyant). Le substantif éternité est absent de ces vers, tandis que se pose le problème de l'exploitation des autres substantifs complétés ou compléments, de l'assignation de leurs adjectifs épithètes respectifs, et de la banalité de la plupart de ces mots pris isolément, qui valent par leur seul agencement syntaxique et tropique.

Le cas de ces "épithètes" en forme d'amples périphrases est fréquent. Dans la mesure où les syntagmes nominaux qui les composent sont eux-mêmes riches en adjectifs, La Porte pouvait choisir d'assigner ceux-ci soit au référent non exprimé de la périphrase, soit au substantif exprimé dans le syntagme périphrastique. Par exemple ${ }^{69}$, la périphrase des poissons, troupes ondoyantes ${ }^{70}$, détermine à l'article " Poisson » l'adjectif verbal ondoiant mais trouve aussi un écho à l'article «Troupe ou Troupeau » avec l'adjectif relationnel poissonneuse ; la périphrase des terres, la maison des humains miserables ${ }^{71}$, se répartit entre l'article «Terre", pour la périphrase maison des humains, et l'article « Humains», pour l'adjectif miserables; la périphrase de Saturne, « vieillard venerable $»^{72}$, donne lieu à deux adjectifs, vieillard et venerable, à l'article "Saturne", et à l'adjectif venerable à l'article "Vieil ou Vieillart». Il nous fallait ainsi prévoir tous les cas possibles, incluant une multitude de cas intermédiaires de fragmentation, de reformulation et de recomposition, en décomposant les périphrases. Cela n'était pas réalisable avec le système de stylage de caractères dans un traitement de texte que nous avions adopté et demandait de reprendre les épithètes polylexicales en postanalyse dans le document XML. Ainsi, pour l'épithète de la terre, la maison des humains misérables, cf. figure 12, nous observons une segmentation de l'épithète dans le corpus balisé par conversion de stylage. Il faut alors revoir les limites de l'épithète enchâssante et, pour une meilleure adéquation descriptive, annoter, dans la balise ouvrante des éléments qui les contiennent, les relations de qualification internes au texte. 


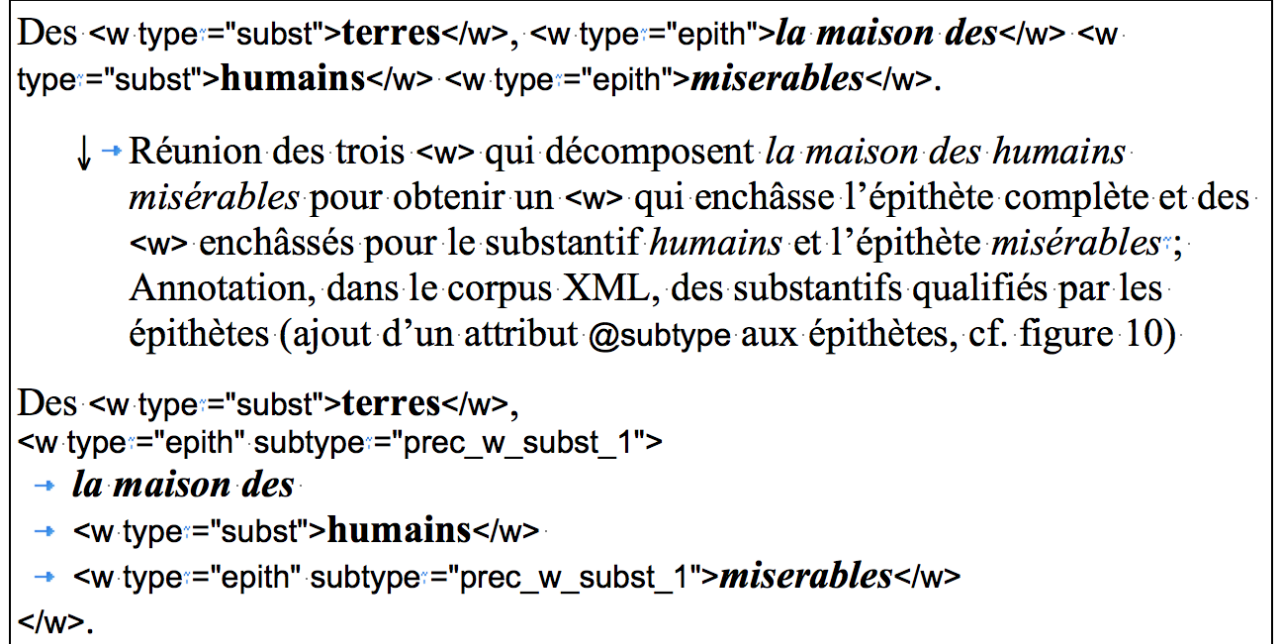

Une autre série de difficultés tient plus nettement encore à l'interprétation du lexicographe. Celui-ci décide en effet parfois d'assigner les épithètes relevées à d'autres substantifs qui lui paraissent plus appropriés, en fonction du sens, ou d'une spécialisation sémantique mise en place dans l'épithétaire, voire de choix idéologiques. Il lui arrive aussi d'étendre à d'autres substantifs parasynonymes les attributions d'épithètes faites à partir du relevé, par exemple aux articles « Or » et «Argent » à partir de l'Hymne de l'or, cf. figure 13,

Figure 13 : Repérage (sous forme d'indices postposés) des articles des Epithetes au sein desquels figurent les épithètes de l'Hymne de l'Or, p. 193-194, v. 317-325

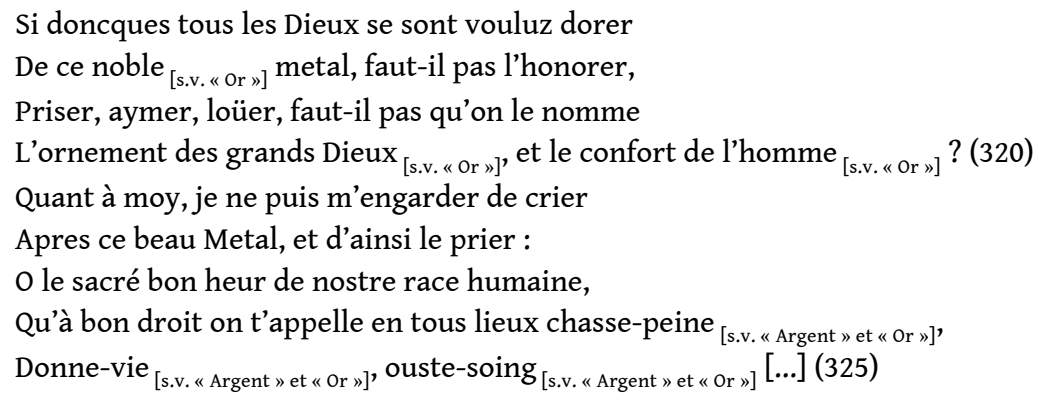

ou de redistribuer les épithètes relevées entre le relevé proprement dit et le commentaire de l'article correspondant, ce qui implique de tenir compte aussi des épithètes insérées dans les commentaires de certains articles et non seulement des listes d'épithètes (alors que nous les avons pour le moment exclues de notre balisage dictionnairique, cf. § 2.1.).

\subsection{Exploiter les extractions faites pour enrichir le texte dictionnairique xmlisé : les pièces bachiques de Ronsard, Baîf et Magny}

Dans un second temps, nous avons structuré le texte ressaisi du dictionnaire de La Porte (cf. § 2.1.) et, après standardisation orthographique, nous sommes attachées à exploiter les extractions faites et la structuration XML du texte dictionnairique pour enrichir ce dernier des attestations observées dans les textes de la Pléiade dépouillés. Le corpus choisi pour cette étude était constitué par les pièces bachiques de la Pléiade, témoignage d'un engouement collectif où se sont illustrés plusieurs poètes de ce groupe dans les 
années 1552-1556. Propices à des envolées litaniques débridées, marquées par l'inventivité verbale, ces pièces permettaient de tester notre outil à travers la confrontation de plusieurs corpus spécialement riches, et à partir d'un seul article de l'épithétaire, « Bacche ou Bacchus $»^{73}$.

\subsubsection{Enrichissements dictionnairiques à partir de relevés}

Cette nouvelle extraction s'est faite par dépouillement manuel et annotation de l'analyse, de manière à préciser les nature et fonction grammaticales, et s'il y avait lieu la valeur rhétorique, de l'épithète de chaque cooccurrence relevée (ce qui fournit les informations de l'élément <interpGrp>cf. § 2.1.). Nous en voyons en figure 14 un tableau de relevé. Le passage litanique ici traité, riche en épiclèses du dieu qui lui sont adressées en liste dans une longue apostrophe oratoire, accumule les épithètes calquées du grec et du latin, que ce soit sous la forme d'hellénismes à peine francisés (Protogone, Evastire, Triete), d'emprunts anciens et déjà bien attestés (Satyre, Martial) ${ }^{74}$, de dérivés sur base savante ( Agnien, Evien, Omadien), de composés français à l'imitation de formes grecques ( Doublecorne, Oeiltoreau, Portelierre, Rompsoucy), ou de traduction par des termes français bien attestés (Père).

Figure 14 : Tableau de relevé des cooccurrences attestées (cf. Lm, t. V, p. 58, v. 73-82)

\begin{tabular}{|c|c|c|c|c|c|c|c|c|}
\hline substantif & épithète & substantif std & épithète std & nature & fct gramm. & fct rhéto. & référence & citation \\
\hline & Pere & Bacchus & père & substantif & & antonomase & Lm V 58, v. 73 & re, Satyre \\
\hline cchus) & Satyre & Bacchus & satyre & subs & the & anto & Lm V & Evoé, \\
\hline (Bacchus) & Protogone & Bacchus & protogone & adiec & apostrophe & antonomase & Lm V 58, v. 74 & Protogone, Evastire \\
\hline (Bacchus) & Evastire & Bacchus & évasti & tif & apostrophe & antonomase & Lm V 58, v. 74 & Protogone, Evastire \\
\hline & ne & & & & & & & \\
\hline cchus) & Agnien & Bacchus & as & & he & anto & $\mathrm{Lm}$ & Doub \\
\hline (Bacchus) & 0 & $\mathrm{Ba}$ & & & & ant & , v. 76 & Oeiltoreau, Ma \\
\hline (Bacchus) & M & & & & & & & Evien \\
\hline & & & & & & & & \\
\hline (Bacchus) & Portelierre & B & p & ac & ohe & ase & v. 77 & Portelierre, Omadien, Triete \\
\hline (Bacchus) & Oma & & om & & apo & & & Portelierre, Omadien, Triete \\
\hline & Triete & & triè & & apostrophe & ant & v. 77 & Portelierre, Omadien, Triete \\
\hline us) & msoucy & cchus & mpt-s & jectif & apostrophe & antonomase & Lm V 59, v. 82 & / Rompsoucy \\
\hline
\end{tabular}

Dans la visée d'une édition scientifique et d'une étude parallèle des expansions nominales dans la poésie de la Pléiade, ce travail de relevé présente un intérêt littéraire, puisqu'il permet d'apprécier chaque cooccurrence au fil du texte et d'en dégager les enjeux poétiques. Il présente en revanche une limite : même si le relevé de chaque cooccurrence et du ou des vers de son attestation référencé(s) permet d'enrichir l'épithétaire de contextes courts qui peuvent être présentés (le contenu des éléments $<q>$ des citations référencées), cf. figure 15, il ne permet pas d'offrir aux lecteurs des contextes longs, sauf dans les cas favorables où plusieurs vers consécutifs ont été relevés pour plusieurs cooccurrences voisines. Et en même temps il montre tout l'intérêt de ces accès à des contextes longs pour se faire une bonne représentation des sources. L'un des objectifs de notre travail serait, à terme, de pouvoir procéder à ces affichages de contextes longs, comme ceux schématisés en figure 16 pour l'article «Bacche ou Bacchus » limité à sa liste d'épithètes (cf. figure 6).

Figure 15 : Prototype d'affichage de la liste d'épithètes de l'article « Bacche ou Bacchus » enrichie de citations courtes référencées

Bacche ou Bacchus.

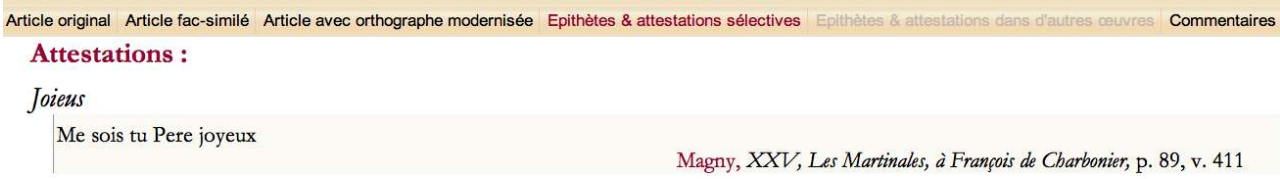


[...]

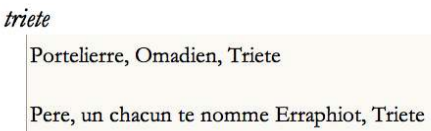

$[\ldots]$

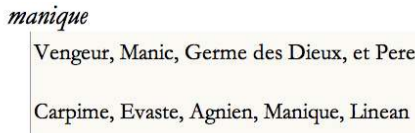

$[\ldots]$

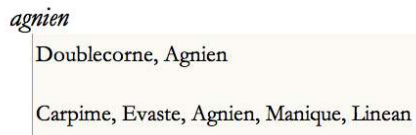

Ronsard, Dithyrambes, Lm V 58, v. 77 Ronsard, L'Hinne de Bacus, Lm VI 184, v. 165 Ronsard, Dithyrambes, Lm V 69, v. 274

Ronsard, L'Hinne de Bacus, Lm VI 188, v. 232

Figure 16 : Schéma d'affichage de l'article «Bacche ou Bacchus » limité à sa liste d'épithètes et enrichi de liens de chacune vers les contextes longs

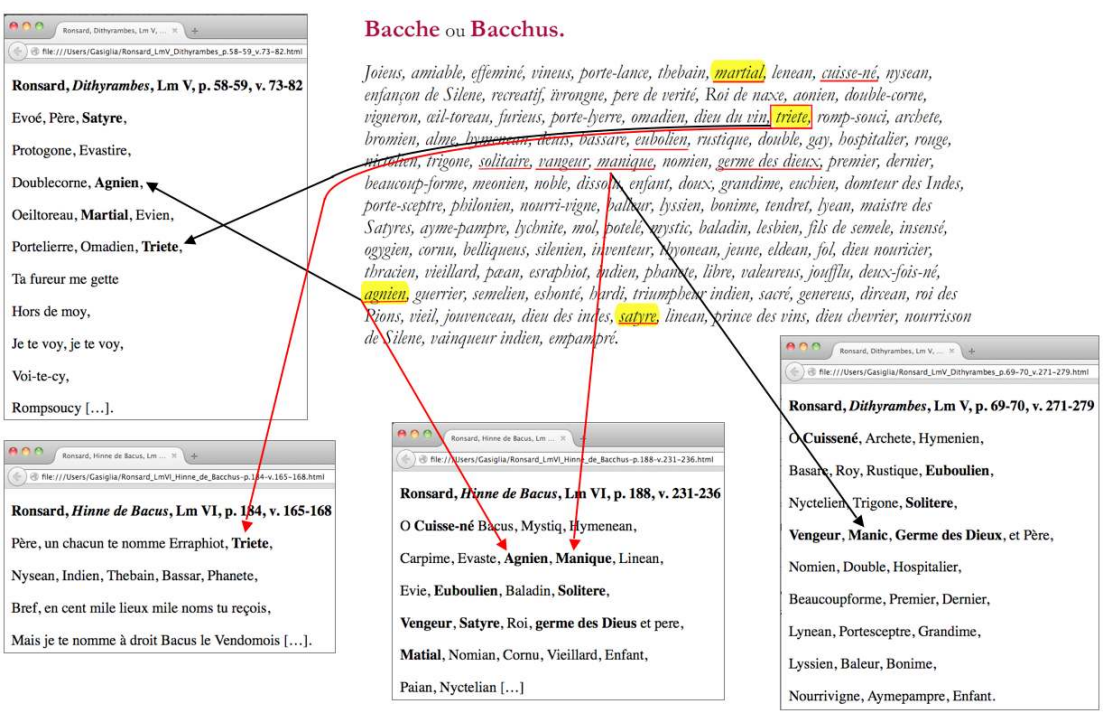

Afin d'offrir de tels accès à des contextes longs, pour les textes dépouillés non disponibles en version électronique, il ne semble pas y avoir de solution autre que la numérisation des sources pertinentes. Pour les autres, il convient d'apprécier comment rendre les sources électroniques exploitables et interopérables avec les relevés déjà réalisés ${ }^{75}$.

\subsubsection{Premiers résultats d'analyse stylistique permis par cet enrichissement dictionnairique}

Le travail d'enrichissement et de confrontation d'ores et déjà mené sur ce corpus, à partir des contextes courts et, expérimentalement, de contextes plus longs a montré son utilité pour l'analyse stylistique, en fournissant des éléments sur la stratification chronologique du relevé effectué par La Porte à partir des différentes pièces bachiques du corpus, et en mettant en évidence des différences de traitement significatives chez Ronsard, Baïf ou Magny.

En ce qui concerne les strates identifiables du relevé effectué par le lexicographe, ces pièces, qui s'imitent les unes les autres, confirment une certaine tendance du 
lexicographe à disposer en désordre les éléments des listes exploitées, mais révèlent aussi différentes étapes qui semblent s'être succédé, suivant les différentes parutions, probablement à partir des Dithyrambes parus dans le recueil anonyme des Folastries, qui était entouré d'un parfum de scandale, et jamais réédités ensuite ${ }^{76}$.

La confrontation systématique des relevés d'épithètes pièce par pièce et auteur par auteur a par ailleurs décuplé les possibilités d'une analyse manuelle en soulignant les prédilections stylistiques des auteurs autant que leurs influences mutuelles et stratégies de réemploi. La comparaison des Bacchanales de Ronsard (1552) et des Martinales de Magny $(1554)^{77}$, deux pièces encore relativement pauvres en épithètes, atteste ainsi déjà le goût de Ronsard pour les épithètes savantes (eldéan, thébain, roi de Naxe, évien ou évan), tandis que Magny insiste davantage sur les aspects festifs (dieu de la vigne, joyeux) : la plupart de ses qualifications mythologiques (père libre, dieu deux fois né, bromien, enfant aux ongles dorés) ne sont guère déconcertantes (à l'exception de bromien, voire de libre, qui joue sur l'épiclèse latine $L i b e r)^{78}$, et il marque une certaine prédilection pour les syntagmes périphrastiques qui sont autant d'expansions du nom père (père indien, père libre, père joyeux), que Ronsard utilise d'ailleurs également (thébain père, père évien) ${ }^{79}$. Les Martinales de Magny s'inspirent également des Dithyrambes de Ronsard, pour deux épithètes (bromien et roi vainqueur) $)^{80}$, et peut-être pour l'apostrophe père, qui est récurrente dans les Dithyrambes.

La confrontation des Dithyrambes de Ronsard à la pièce du même nom composée par Baiff ${ }^{81}$ , deux pièces en vers libres composées en 1553 à l'occasion d'une fête en l'honneur de Jodelle, met en évidence un contraste flagrant entre les litanies de ces pièces. Baïf emploie presque exclusivement des syntagmes antonomasiques formés de dieu et d'une épithète, et des épithètes composées au sens plutôt quotidien (foudre-né, donne-bonheur, brise-souci, doux-ravissant, démon aime-danse). Il est notable que La Porte n'a repris aucune de ces épithètes ${ }^{82}$. Les Dithyrambes de Ronsard donnent lieu en revanche à une véritable explosion litanique, liée au mimétisme de la "fureur ». Les épithètes en sont savantes, gréco-latines (dyphien, cryphien, protogone...), inspirées des Hymnes orphiques et du poète néolatin Marulle ${ }^{83}$, et les épithètes composées calquent la structure des épithètes composées grecques (double-corne, œeil-taureau, beaucoup-forme à côté de rompt-souci ou aime-pampre, plus proches de la pratique de Baïf).

Lorsque, enfin, Ronsard récrit les Dithyrambes dans l'Hinne de Bacus (1555) ${ }^{84}$, apparaissent des différences liées à la structure hymnique du texte, composé cette fois en alexandrins, et à son enrichissement mythologique, car il affiche une tournure savante. L'Hinne comporte, comme dans les Dithyrambes, deux litanies, mais à la différence de ce qui se produit dans les Dithyrambes, la première consiste en un exposé des noms divins, avant que la deuxième n'imite la «fureur » poétique. Ainsi, Ronsard recompose les listes, tant dans le choix des mots que sur le plan métrique, et c'est ainsi qu'il en viendra à apparaître comme le plus à même d'exprimer l'« enthousiasme " antique ${ }^{85}$ et comme le médiateur par excellence de l'inspiration antique dans le corpus bachique en France et en Europe.

De fait, La Porte a principalement exploité Ronsard. L'article « Bacche ou Bacchus » limité à sa liste d'épithètes enrichie d'indications d'auteurs et de volumes sources de l'édition de P. Laumonier (cf. figure 6) le montre nettement. Une épithète du relevé ne se trouve que chez Baïf (sémélien), posant la question de son influence aussi pour d'autres épithètes, qui se trouvent également chez Ronsard (nictolien / nyctélien) et que La Porte relève parmi d'autres épithètes issues des Dithyrambes de Ronsard ${ }^{86}$. Quelques épithètes (peu 
significatives) se trouvent également chez Magny ${ }^{87}$. Mais la présence de Ronsard est en tout cas écrasante.

Les jeux de différenciation que nous avons pu observer au sein de la jeune Pléiade témoignent ainsi de la réalité de styles différents tant en termes d'appréhension de la "fureur» que de poétiques de la qualification, selon que l'accent était mis sur une inspiration délirante, censée libérer le langage, ou savante, traduisible par une mythologie moins débridée, prenant appui sur le concept (dans les expansions des noms roi, père, etc.), ou encore plus familière. La poétique de Ronsard, caractérisée par l'essor des litanies savantes, explique que celui-ci ait été perçu comme le représentant par excellence d'une poétique associant la «fureur » poétique à l'enrichissement lexical : c'est cette reconnaissance que traduisent les relevés de La Porte.

\section{Conclusion}

Par ce retour sur les dépouillements entrepris dans le cadre de cette édition des Epithetes de La Porte, qui ambitionne d'identifier les sources et les choix de ce lexicographe, nous souhaitions faire connaître cet ambitieux projet et exposer comment un rapprochement de cooccurrents lexicaux entre textes $\mathrm{du} \mathrm{xVI}^{\mathrm{e}}$ siècle peut conduire à s'interroger de diverses manières sur l'état de la langue française de cette époque (son orthographe, les relations synonymiques entretenues par des substantifs ou syntagmes nominaux lexicalisés, les éventuelles relations dérivationnelles perceptibles entre différentes formes d'épithètes, etc.) et à corréler ces approches à des prétraitements des données à manipuler, afin d'effectuer les appariements de cooccurrences relevées par La Porte et attestées dans les textes de la Pléiade explorés. Le travail effectué au Xvi siècle pour Les Epithetes a des liens avec ce qui constitue la documentation en corpus de la lexicographie moderne, le dépouillement de textes à la recherche de ce qui rendra le mieux compte de l'usage leur est commun. Les dépouillements faits pour le Trésor de la langue française ${ }^{88}$ ou pour les dictionnaires, en particulier britanniques, qui offrent des citations ou des énoncés attestés (ou à peine révisés) comme exemples d'emplois, partagent le recours aux corpus, même si pour La Porte il n'était évidemment pas électronique. Mais l'intérêt particulier de l'étude des relevés des Epithetes consiste justement dans ce que le lexicographe a apporté comme part interprétative, ce que les rédacteurs modernes ne font habituellement pas.

\section{BIBLIOGRAPHIE}

Sources primaires

Baif, Jean-Antoine de, CEuvres complètes, I, Euvres en rime, $\mathrm{I}^{\mathrm{re}}$ partie, Neuf Livres des Poemes, éd. J. Vignes (dir.) et al., Paris, Champion, 2002.

Besly, Jean, commentateur de Ronsard, voir Ronsard.

Dorat, Jean, traducteur de Ronsard, voir Ronsard. 
Du Bellay, Joachim, La Deffence et illustration de la langue françoyse, éd. J.-Ch. Monferran, Genève, Droz, 2001.

Du Bellay, Joachim, CEuvres poétiques, éd. H. Chamard, Paris, Nizet, 6 vol., 1908-1931.

Dupuys, Jacques, voir Nicot.

Erasme, Desiderius, Adages, éd.-trad. J.-C. Saladin (dir.) et al., Paris, Belles Lettres, $2^{\mathrm{e}}$ éd., 2013, 5 vol.

Érasme, Desiderius, De copia verborum ac rerum, ASD (Opera omnia, Amsterdam, North-Holland Publishing Company), t. I-6, éd. B. I. Knott, 1988.

Estienne, Robert, Dictionarium seu Latinae linguae Thesaurus, Paris, R. Estienne, $1531 ; 2^{\mathrm{e}}$ éd., 1536.

Estienne, Robert, Dictionarium latinogallicum, Paris, R. Estienne, $1538 ; 2^{\mathrm{e}}$ éd., 1546.

Estienne, Robert, Dictionaire francoislatin, Paris, R. Estienne, 1539 ; rééd. Paris, R. Estienne, 1549. Rééd. suivantes : voir Thierry, Nicot et Dupuys.

La Porte, Maurice de, Les Epithetes [1571], fac-similé, Genève, Slatkine reprints, 1973.

La Porte, Maurice de, Les Epithetes [1571], éd. F. Rouget, Paris, Champion, 2009.

La Porte, Maurice de, Les Epithetes [1571], éd. partielle en ligne : http://www.preambule.net/.

Magny, Olivier de, Les Gayetez [1554], éd. A. R. Mac Kay, Genève, Droz, 1968.

Marulle (Marullo, Michele), Hymnes naturels, éd.-trad. J. Chomarat, Genève, Droz, 1995.

Muret, Marc-Antoine de, commentateur de Ronsard, voir Ronsard.

Nicot, Jean, et Dupuys, Jacques, Dictionaire françois-latin, Paris, Dupuys et [Lyon], de Hus, $1573 ; 2^{\mathrm{e}}$ éd. $1584 ; 3^{\mathrm{e}}$ éd. 1585 . Voir Estienne.

Nicot, Jean, Thresor de la langue françoyse, Paris, D. Douceur, 1606 ; éd en ligne (cf. ARTFL Project, Université de Chicago) : https://artfl-project.uchicago.edu/content/advanced-search.

Ravisius Textor (Tixier de Ravisy, Jean), Specimen Epithetorum..., Paris, H. Estienne pour R. Chaudière, 1518.

Ravisius Textor (Tixier de Ravisy, Jean), Epitheta..., Paris, R. Chaudière, 1524.

Ronsard, Pierre de, et Dorat, Jean, Hymne de Bacus, par Pierre de Ronsard, avec la version latine de Jean Dorat, Paris, A. Wechel, 1555.

Ronsard, Pierre de, Les Euvres de P. de Ronsard, Paris, N. Buon, 1604, 10 t. en 5 vol., t. VII, Les Hynnes, avec commentaire de J. Besly.

Ronsard, Pierre de, Cuures complètes, éd. P. Laumonier, Paris, STFM, 1914-1975, 18 t. en 20 vol. Ronsard, Pierre de, Cuuvres complètes, éd. J. Céard, D. Ménager et M. Simonin, Paris, Gallimard, «Bibliothèque de la Pléiade », 1993-1994, 2 vol.

Ronsard, Pierre de, Les Amours et Les Folastries (1552-1560), éd. A. Gendre, Paris, LGF, 1993.

Ronsard, Pierre de, \& Muret, Marc-Antoine de, Les Amours, leurs Commentaires [1553], éd. C. de Buzon et P. Martin, Paris, Classiques Didier Érudition, 1999.

Smyters, Anthoni, Epitheta..., Rotterdam, Jan van Waesberghe, 1620 ; éd. modernisée Het versierde woord. De Epitheta of woordcombinaties uit 1620, éd. N. van der Sijs, Amsterdam-Anvers, Contact, 1999. URL : http://www.dbnl.org/tekst/sijs002vers01_01/.

Thierry, Jean, Dictionaire francoislatin, Paris, Dupuys \& Macé, 1564. Voir Estienne. 
Ouvrages critiques

Antidote mobile - Dictionnaires \& guides (2011), application pour iPhone/iPod touch/iPad. Montréal, Druide informatique ; version 1.5.5. 2014.

Beauchesne, Jacques, Dictionnaire des cooccurrences, Montréal, Guérin, 2001.

Beaulieux, Charles, «L'orthographe de Ronsard », Mélanges Henri Chamard, Paris, Nizet, 1951, p. 125-135.

Berlan, Françoise, «Épithète grammaticale et épithète rhétorique », Cahiers de lexicologie, 39, 1981, II, p. 5-23.

Berlan, Françoise, «L'épithète entre rhétorique, logique et grammaire aux XVII ${ }^{\mathrm{e}}$ et XVIII ${ }^{\mathrm{e}}$ siècles ", Histoire, Épistémologie, Langage, XIV/1, 1992, L'adjectif : perspectives historique et typologique, dir. B. Colombat, p. 181-198.

Bierbach, Mechtild, «Les Épithetes de Maurice de La Porte de 1571 : ouvrage lexicographique, encyclopédique et rhétorique ", Actes $d u$ XVIII ${ }^{e}$ Congrès international de linguistique et de philologie romanes, dir. D. Kremer, Tübingen, M. Niemeyer, 1989, t. IV-6, p. 44-60.

Brunot, Ferdinand, Histoire de la langue française des origines à nos jours, t. II, Le XVI siècle, Paris, A. Colin, 1967.

Catach, Nina, L'Orthographe [1978], Paris, PUF, 2011.

Cave, Terence, Cornucopia. Figures de l'abondance au XVI siècle : Erasme, Rabelais, Ronsard, Montaigne [1979], trad. fr. G. Morel, Paris, Macula, 1997.

Charest, Simon, Fontaine, Jean, et Saint-Germain, Jean, Le grand Druide des cooccurrences, Montréal, Éditions Druide, 2012. Voir Antidote mobile - Dictionnaires \& guides (2011).

Chevalier, Jean-Claude, «Quelques remarques sur un historique cavalier de la synonymie », Synonymie et «differentice» : théories et méthodologies de l'époque classique à l'époque moderne, éd. M. G. Adamo et P. Radici Colace, Messine-Naples, 2006, p. 27-42.

Chomarat, Jacques, Grammaire et rhétorique chez Érasme, Paris, Belles Lettres, 1981, 2 vol., 1980-1981.

Emmi, Yoshito, « Détermination de l'année de composition de chaque sonnet des Amours (1552), d'après l'orthographe ", Revue des Amis de Ronsard, 2, 1989, p. 1-18.

Ford, Philip, De Troie à Ithaque : réception des épopées homériques à la Renaissance, Genève, Droz, 2007. Gasiglia, Nathalie, « Le lexicographe au travail... », 2015, voir Pouey-Mounou, Anne-Pascale. Gasiglia, Nathalie, et Pouey-Mounou, Anne-Pascale, « La Porte, la Pléiade et Bacchus », Inqualifiables fureurs. Les qualifications des figures de l'inspiration à la Renaissance, éd. M. Huchon et A.-P. Pouey-Mounou, Paris, Classiques Garnier, à paraître.

Hache, Sophie et Pouey-Mounou, Anne-Pascale (éd.), L'épithète, la rime et la raison. La lexicographie poétique en Europe, $\mathrm{XVI}^{e}-\mathrm{XVII}{ }^{e}$ siècles, Paris, Classiques Garnier, 2015.

Huchon, Mireille, « Ronsard et les 'monstres' de l'écriture : 'en attendant meilleure reformation' ", Ronsard, la Trompette et la Lyre, catalogue, Paris, BnF, 1985, p. 170-172. Hummel, Pascale, L'épithète pindarique. Étude historique et philologique, Berne, P. Lang, 1999. Istasse, Nathaël, « Le Specimen Epithetorum (1518) et les Epitheta (1524). J. Ravisius Textor compilateur et créateur ", L'épithète, la rime et la raison. La lexicographie poétique en Europe, $\mathrm{XVI}^{e}-\mathrm{XVII}{ }^{e}$ siècles, éd. S. Hache et A.-P. Pouey-Mounou, Paris, Classiques Garnier, 2015, p. 79-121. 
Laurent, Nicolas, « Les rapports entre le nom propre et le nom commun dans la réflexion sur l'antonomase aux XVI ${ }^{\mathrm{e}}$ et XVII ${ }^{\mathrm{e}}$ siècles ", Le Français préclassique (1500-1650), 16, 2014, p. 67-79.

Le Fur, Dominique (dir.), Dictionnaire des combinaisons de mots, Paris, Le Robert, 2007.

Leclercq, Odile, Construction d'un savoir et d'un savoir-faire dans le traitement du lexique français aux XVI et XVII ${ }^{e}$ siècles, thèse, Univ. Paris VII-Denis Diderot, dir. F. Mazière, 2006.

Maira, Daniele, «'L'encre et la voix' : Muret et la fabrique des Amours de Ronsard », BHR, 72-3, 2010, p. 621-639.

Pédeflous, Olivier, « L'atelier du poète-lexicographe au début du XVI ${ }^{\mathrm{e}}$ siècle en France », Camenae, 1, janv. 2007. URL : http://www.paris-sorbonne.fr/IMG/pdf/Olivier_Pedeflous_definitif.pdf.

Pouey-Mounou, Anne-Pascale, « Grandeur et décadence d'un tout petit genre : les épithétaires de la Renaissance ", Esculape et Dionysos, Mélanges Jean Céard, Genève, Droz, 2008, p. 1065-1079.

Pouey-Mounou, Anne-Pascale, « Dictionnaires d'épithètes et de synonymes aux XVI ${ }^{\mathrm{e}}$ et XVII ${ }^{\mathrm{e}}$ siècles : du lexique au manuel », BHR, $n^{\circ}$ 75/1, 2013, p. 47-65.

Pouey-Mounou, Anne-Pascale, et Gasiglia, Nathalie, « Le lexicographe au travail. Le traitement des Hymnes cosmologiques de Ronsard (1555-1556) dans les Epithetes de La Porte », L'épithète, la rime et la raison. La lexicographie poétique en Europe, XVI ${ }^{e}-\mathrm{XVII}{ }^{e}$ siècles, éd. S. Hache et A.-P. PoueyMounou, Paris, Classiques Garnier, 2015, p. 237-277.

Pouey-Mounou, Anne-Pascale, « Brocards, pastiches et mélanges : bas morceaux choisis des Epithetes de La Porte », Textes au corps. Promenades et musardises sur les terres de Marie Madeleine Fontaine, Genève, Droz, 2015, p. 129-145.

Pouey-Mounou, Anne-Pascale, « La Porte et les commentaires des Amours », Mélanges Jean Lecointe, éd. C. Gutbub, Cl. La Charité, R. Menini et T. Tran, Paris, Classiques Garnier, à paraître.

Pouey-Mounou, Anne-Pascale, « La Porte, la Pléiade et Bacchus », à paraître, voir Gasiglia, Nathalie.

Quemada, Bernard, Les dictionnaires du français moderne (1539-1863). Étude sur leur histoire, leurs types et leurs méthodes, Paris, Didier, 1968.

Reguig, Delphine, «Froideur et saveur de la rime chez Boileau », L'épithète, la rime et la raison. La lexicographie poétique en Europe, $\mathrm{XVI}^{e}-\mathrm{XVII}{ }^{e}$ siècles, éd. S. Hache et A.-P. Pouey-Mounou, Paris, Classiques Garnier, 2015, p. 367-382.

Rey, Alain (dir.), Dictionnaire historique de la langue française, Paris, Le Robert, 1992.

Rouget, François, Ronsard et le livre. Étude de critique génétique et d'histoire littéraire, II, Les livres imprimés, Genève, Droz, 2012.

Text Encoding Initiative, URL : http://www.tei-c.org/Guidelines/P5/ (recommandations générales) et http://www.tei-c.org/release/doc/tei-p5-doc/fr/html/DI.html (recommandations propres aux dictionnaires).

Trésor de la langue française. Dictionnaire de la langue du XIX ${ }^{e}$ et du XX ${ }^{e}$ siècle (1789-1960), 16 vol., Paris, Éditions du Centre National de la Recherche Scientifique (t. 1-10) / Gallimard (t. 11-16), 1971-1994 ; éd. en ligne : http://atilf.atilf.fr/tlf.htm.

Wooldridge, Terence Russon, Les débuts de la lexicographie française : Estienne, Nicot et le Thresor de la langue françoyse (1606), Toronto, University of Toronto Press, 1977 ; $2^{\mathrm{e}}$ éd. : http:// www.etudes-francaises.net/dossiers/wooldridge_debuts/, 2010. 
Zinglé, Henri, et Brobeck-Zinglé, Marie-Louise, Dictionnaire combinatoire du français. Expressions, locutions et constructions, Paris, La maison du dictionnaire, 2003.

\section{NOTES}

1. Nous remercions les relecteurs d'une première version de ce texte pour les remarques et suggestions formulées.

2. La Porte, Les Epithetes [1571], fac-similé, Genève, Slatkine reprints, 1973. Il existe de ce dictionnaire une éd. papier sans annotation scientifique systématique des sources dans le détail du texte (éd. F. Rouget, Paris, Champion, 2009), ainsi qu'une éd. partielle en ligne (en cours) offrant des liens vers d'autres occurrences des épithètes relevées mais sans vocation à identifier les sources du recueil (http://www.preambule.net/). Pour une mise en perspective historique de ce genre parmi l'essor de la lexicographie au xvI ${ }^{\mathrm{e}}$ siècle, voir B. Quemada, Les dictionnaires du français moderne (1539-1863). Étude sur leur histoire, leurs types et leurs méthodes, Paris, Didier, 1968 ; O. Leclercq, Construction d'un savoir et d'un savoir-faire dans le traitement du lexique français aux $\mathrm{XVI}^{e}$ et XVII ${ }^{e}$ siècles, thèse, 2006, Univ. Paris VII-Denis Diderot, dir. F. Mazière ; A.-P. Pouey-Mounou, "Grandeur et décadence d'un tout petit genre : les épithétaires de la Renaissance », Esculape et Dionysos, Mél. J. Céard, Genève, Droz, 2008, p.1065-1079; et L'épithète, la rime et la raison. La lexicographie poétique en Europe, $\mathrm{XVI}^{e}-\mathrm{XVII} \mathrm{e}^{e}$ siècles, éd. S. Hache et A.-P. Pouey-Mounou, Paris, Classiques Garnier, 2015.

3. Nous citons Ronsard dans Euvres complètes, éd. P. Laumonier, Paris, STFM, 1914-1975, 18 t. en 20 vol. [désormais : Lm].

4. La Porte, Les Epithetes, op. cit., « Advertissement au lecteur ».

5. J. Beauchesne, Dictionnaire des cooccurrences, Montréal, Guérin, 2001.

6. H. Zinglé et M.-L. Brobeck-Zinglé, Dictionnaire combinatoire du français. Expressions, locutions et constructions, Paris, La maison du dictionnaire, 2003.

7. D. Le Fur (dir.), Dictionnaire des combinaisons de mots, Paris, Le Robert, 2007.

8. S. Charest, J. Fontaine et J. Saint-Germain, Le grand Druide des cooccurrences, Montréal, Éditions Druide, 2012. Ce dictionnaire est également consultable dans l'application dictionnairique: Antidote mobile - Dictionnaires \& guides (2011), application pour iPhone/iPod touch/iPad. Montréal, Druide informatique ; version 1.5.5. 2014.

9. T. R. Wooldridge, Les débuts de la lexicographie française : Estienne, Nicot et le Thresor de la langue françoyse (1606), Toronto, University of Toronto Press, 1977; $2^{\mathrm{e}}$ éd., http://www.etudesfrancaises.net/dossiers/wooldridge_debuts/, 2010. Cf. T. R. Wooldridge ( ibid., § «Matières préfatoires » / "Structure du document ») : «Depuis la publication en 1977 de la première édition des Débuts de la lexicographie française, le Thresor de la langue françoyse a été informatisé dans les années 1980 [...]. Aussi la deuxième édition a pu profiter du plein accès au texte offert par la version électronique du Thresor [...] Les conclusions de la première édition restent toujours valables; nous avons simplement remplacé, à divers endroits du texte, des échantillons par des listes exhaustives [...]».

10. Cf. ibid. § « Avant-propos».

11. Ibid.

12. Cf. ibid. $\S$ «1. Du dictionnaire latin au dictionnaire français», en nous limitant à ce qui permet de situer les ouvrages contemporains des Epithetes :

- tout commence avec le Dictionarium seu Latinae linguae Thesaurus de Robert Estienne (Paris, R. Estienne, $1531 ; 2^{\mathrm{e}}$ éd., 1536);

- des deux éditions de ce dictionnaire de latin, R. Estienne dérive un bilingue latin / français, le Dictionarium latinogallicum (Paris, R. Estienne, $1538 ; 2^{\mathrm{e}}$ éd., 1546) ; 
- de l'édition de 1538 de ce dernier, R. Estienne dérive un dictionnaire français / latin, le Dictionaire francoislatin (Paris, R. Estienne, 1539), cette dérivation se faisant par "retournement de nomenclature", c'est-à-dire que les items latins, qui constituaient les nomenclatures du Dictionarium latinogallicum et qui étaient traités - traduits ou expliqués - en français, deviennent, dans le Dictionaire francoislatin, les traductions des anciennes séquences traductionnelles ou explicatives, elles-mêmes devenues les items traités ;

- le Dictionaire francoislatin est réédité, sous le même titre, par R. Estienne (Paris, R. Estienne, 1549) avec des enrichissements issus de l'édition de $1546 \mathrm{du}$ Dictionarium latinogallicum, puis par Jean Thierry (Paris, Dupuys \& Macé, 1564);

- la réédition suivante a été proposée par Jean Nicot et Jacques Dupuys en 1573 (et réimprimée en 1584 et 1585 ) sous le titre de Dictionaire françois-latin (Paris, Dupuys et [Lyon], de Hus, $1573 ; 2^{\mathrm{e}}$ éd. $1584 ; 3^{\mathrm{e}}$ éd. 1585) ;

- et c'est de ce dernier dictionnaire que dérive le Thresor de la langue françoyse de Jean Nicot (Paris, D. Douceur, 1606).

13. Cf. ibid. $\S$ «2.2.2. Délimitation sémantique ».

14. Pour cela, nous exploitons le texte du Thresor de la langue françoyse de la section consacrée aux « Dictionnaires d'autrefois » du site ARTFL Project (Université de Chicago, cf. https://artflproject.uchicago.edu/content/advanced-search). Nous y copions les articles "Amour», mais aussi « Amoureux », « Amoureusement » et « Amourachement », puisque leurs textes figurent s.v. «Amour» dans les éditions de 1564 et 1606.

15. Cf. T. R. Wooldridge (2010, § « 2.2.7.2.1. Indications grammaticales et accentuelles »).

16. Ces 66 combinaisons en 1564 (ou 67 en 1606) contiennent respectivement amour (52 ou 53), amoureux (10), amoureuse (2), amoureusement (1) ou amourachement (1).

17. Cf. n. 12 .

18. Wooldridge (2010, $\S \ll 2.2 .8 .1 .1$. Informations ») indique que les articles du Thresor de la langue françoyse proposent divers composants : «Adresse [...] Variantes [...] Remarque sur l'orthographe de l'adresse [...] Remarque sur la prononciation de l'adresse [...] Indication accentuelle : place de l'accent ou nombre de syllabes [...] Étymologie [...] Dérivés et 'cognates' [...] Catégorisation grammaticale [...] Définition [...] Exemples d'emploi : syntagmes lexicalisés ou libres [...] Équivalents latins de l'adresse / d'un exemple [...] Équivalents non latins de l'adresse / d'un exemple [...] Remarques d'usage: fonctionnement temporel / spatio-linguistique / socioprofessionnel / stylistique / quantitatif [...] Citations: autonymes, encyclopédiques [...] Commentaires encyclopédiques : métamétalinguistiques [...], extra-linguistiques [...] Renvois [... ]».

19. Voir J.-Cl. Chevalier, "Quelques remarques sur un historique cavalier de la synonymie", Synonymie et "differentice": théories et méthodologies de l'époque classique à l'époque moderne, éd. M. G. Adamo et P. Radici Colace, Messine-Naples, 2006, p. 27-42.

20. Ravisius Textor, Specimen Epithetorum..., Paris, H. Estienne pour R. Chaudière, 1518, et Epitheta..., Paris, R. Chaudière, 1524. Voir O. Pédeflous, «L'atelier du poète-lexicographe au début du $\mathrm{XVI}^{\mathrm{e}}$ siècle en France", Camenae, 1, janv. 2007 (http://www.paris-sorbonne.fr/IMG/pdf/ Olivier_Pedeflous_definitif.pdf), et N. Istasse, «Le Specimen Epithetorum (1518) et les Epitheta (1524). J. Ravisius Textor compilateur et créateur ", L'épithète, la rime et la raison, op. cit., p. 79-121.

21. Voir B. Quemada, Les Dictionnaires du français moderne, op. cit., p. 100-101. M. Bierbach met en garde contre une perspective anachronique: «Les Épithetes de Maurice de La Porte de 1571: ouvrage lexicographique, encyclopédique et rhétorique ", Actes $d u$ XVIII ${ }^{e}$ Congrès international de linguistique et de philologie romanes, dir. D. Kremer, Tübingen, M. Niemeyer, 1989, t. IV-6, p. 44-60.

22. Voir Ph. Ford, De Troie à Ithaque: réception des épopées homériques à la Renaissance, Genève, Droz, 2007, chap. 6, p. 275-283.

23. Voir L'épithète, la rime et la raison, op. cit., préface et D. Reguig, «Froideur et saveur de la rime chez Boileau ». 
24. Érasme, De copia, ASD (Opera omnia, Amsterdam, North-Holland Publishing Company), t. I-6, éd. B. I. Knott, 1988, p. 27-32 ; J. Chomarat, Grammaire et rhétorique chez Érasme, Paris, Belles Lettres, 1981, 2 vol. , 1980-1981, t. II, p. 715-760, et T. Cave, Cornucopia. Figures de l'abondance au XVI siècle : Erasme, Rabelais, Ronsard, Montaigne [1979], trad. fr. G. Morel, Paris, Macula, 1997, p. 46-61.

25. Erasme, Adages, éd.-trad. J.-C. Saladin (dir.) et al., Paris, Belles Lettres, $2^{\mathrm{e}}$ éd., 2013, 5 vol.

26. Voir A.-P. Pouey-Mounou, "Grandeur et décadence... », art. cit., et «Dictionnaires d'épithètes et de synonymes aux XVI ${ }^{\mathrm{e}}$ et XVII ${ }^{\mathrm{e}}$ siècles : du lexique au manuel ", BHR, $\mathrm{n}^{\circ} 75 / 1,2013$, p. 47-65.

27. Voir F. Rouget, Ronsard et le livre. Étude de critique génétique et d'histoire littéraire, II, Les livres imprimés, Genève, Droz, 2012, p. 27-29, 47-50; et id., préface à son éd. des Epithetes, éd. cit.

28. Voir J. Du Bellay, La Deffence et illustration de la langue françoyse, éd. J.-Ch. Monferran, Genève, Droz, 2001.

29. A. Smyters, Epitheta..., Rotterdam, Jan van Waesberghe, 1620 ; éd. modernisée Het versierde woord. De Epitheta of woordcombinaties uit 1620, éd. N. van der Sijs, Amsterdam-Anvers, Contact, 1999 (http://www.dbnl.org/tekst/sijs002vers01_01/).

30. Voir P. Hummel, L'épithète pindarique. Étude historique et philologique, Berne, P. Lang, 1999, I ${ }^{\text {re }}$ partie, chap. 3, p. 57-101; F. Berlan, «Épithète grammaticale et épithète rhétorique ", Cahiers de lexicologie, 39, 1981, II, p. 5-23 ; id., " L'épithète entre rhétorique, logique et grammaire aux XVII ${ }^{\mathrm{e}}$ et XVIII ${ }^{\mathrm{e}}$ siècles ", Histoire, Épistémologie, Langage, XIV/1, 1992, L'adjectif: perspectives historique et typologique, dir. B. Colombat, p. 181-198.

31. Voir F. Brunot, Histoire de la langue française des origines à nos jours, t. II, Le XVI e siècle, Paris, A. Colin, 1967, p. 228.

32. D'après Ronsard, Folastries, III, Lm, t. V, v. 106-112 (paroles de la vieille Catin devenue bigote ! ) .

33. Selon les orientations anti-pétrarquistes prises par la Pléiade dans les années 1553-1560 : voir J. Du Bellay, élégie A une dame ( $2^{\mathrm{e}}$ éd. du Recueil de Poesie [1553], Euvres poétiques, éd. H. Chamard, Paris, Nizet, 6 vol. , 1908-1931, t. IV, p. 205-215), réécrite dans l'élégie Contre les petrarquistes ( Divers jeux rustiques [1558], t. V, p. 69-77) ; Ronsard, élégie A son Livre, épilogue de la Nouvelle Continuation des Amours [1556] rééd. comme prologue du Second Livre des Amours à partir de 1560, Lm, t. VII, p. 315-325.

34. Le succès des Epithetes à la fin du XvI ${ }^{\mathrm{e}}$ siècle accrédite l'idée qu'il aurait pu servir lui-même de source à des rédacteurs.

35. Le site "Le Préambule des innombrables" en propose une version qui est en cours d'élaboration et qui offre des enrichissements tout à fait différents de ceux qui font l'objet de notre travail. Cf. n. 2.

36. Pour cette fusion du texte dictionnairique et des citations référencées, un programme spécifique (une transformation XSLT) a été élaboré : pour chaque substantif en adresse associé à chaque épithète de l'article, ces deux items étant considérés avec leur graphie standardisée, la transformation recherche, parmi les citations extraites, référencées et analysées, celles pour lesquelles les cooccurrents enregistrés (attestés ou reconstitués) ont des formes graphiques standardisées identiques et les intègre au texte dictionnairique en respectant le modèle de document XML schématisé en figure 7.

37. Cf. http://www.tei-c.org/Guidelines/P5/.

38. Cf. http://www.tei-c.org/release/doc/tei-p5-doc/fr/html/DI.html.

39. Quand un auteur est mentionné plusieurs fois à la suite d'une épithète, comme l'est Baïf pour semelien (deux fois), ou que Ronsard l'est avec la même indication de volume, comme « Ronsard, Lm VI " l'est (deux fois également) pour cuisse-né ou enfant, c'est que la cooccurrence du substantif en adresse et de l'épithète est attestée plusieurs fois chez le même auteur ou chez Ronsard dans le même volume de l'édition de Laumonier. Un affichage HTML plus abouti du texte 
dictionnairique réduira ces redondances en donnant plutôt les cardinalités en cas d'occurrences multiples.

40. APPM indique que c'est Anne-Pascale Pouey-Mounou qui a proposé ces informations.

41. Il s'agit ici d'éléments d'interprétation qui fournissent la nature grammaticale et les fonctions grammaticale et rhétorique de l'épithète.

42. S.v. "Bacche ou Bacchus ", en figure 8 , « deux fois né » devrait être balisé comme épithète dans le commentaire final. S.v. "Amour ", en figure 1, " gouffre de maux », " rage » et « fumee » devraient l'être dans la citation, comme «foles» dans le premier commentaire s.v. «Amoureuse ou Amante ", en figure 3.

43. Le temps de lecture-correction de ce qui serait fourni par une reconnaissance optique des caractères serait plus long que celui de la ressaisie (incluant le temps de lecture-correction que cette méthode implique également).

44. Cet ensemble de textes ne sera pas repris au §3., quand nous présenterons deux traitements opérés sur d'autres sélections de textes - les Hymnes cosmologiques de Ronsard (§ 3.1.) et les pièces bachiques de Ronsard, Baïf et Magny (\$ 3.2.).

45. Sur l'histoire éditoriale des Amours de 1552-1553, voir D. Maira, «'L'encre et la voix' : Muret et la fabrique des Amours de Ronsard », BHR, 72-3, 2010, p. 621-639. Sur la disposition des recueils d'Amours dans les éd. collectives à partir de 1560, voir Ronsard, Euvres complètes, éd. J. Céard, D. Ménager et M.Simonin, Paris, Gallimard, «Bibliothèque de la Pléiade », 1993-1994, 2 vol. [désormais : éd. Pléiade], t. I, p. 1215-1223.

46. Ronsard \& Muret, Les Amours, leurs Commentaires [1553], éd. C. de Buzon et P. Martin, Paris, Classiques Didier Érudition, 1999 [désormais : Muret 1553]. Voir A.-P. Pouey-Mounou, « La Porte et les commentaires des Amours ", à paraître dans les Mél. J. Lecointe, éd. C. Gutbub, Cl. La Charité, R. Menini et T. Tran, Paris, Classiques Garnier.

47. Voir N. Catach, L'Orthographe [1978], Paris, PUF, 2011, p. 24-29. Ce bref résumé de l'état de la question sur les Amours s'inspire d'un document transmis par J.-Ch. Monferran. Voir aussi Ch. Beaulieux, "L'orthographe de Ronsard », Mél. H. Chamard, Paris, Nizet, 1951, p. 125-135, et M. Huchon, «Ronsard et les 'monstres' de l'écriture : 'en attendant meilleure reformation'", Ronsard, la Trompette et la Lyre, catalogue, Paris, Bibl. Nationale, 1985, p. 170-172.

48. Voir par ex. des tentatives comme celle de Y.Emmi, "Détermination de l'année de composition de chaque sonnet des Amours (1552), d'après l'orthographe ", Revue des Amis de Ronsard, 2, 1989, p. 1-18.

49. Ronsard, Lm, t. IV, p. 5, v. 5 ; Muret 1553, p. 15 ; et La Porte, Les Epithetes, s.v. « Ieunesse».

50. Ronsard, Lm, t. IV, p. 6, v. 13 ; Muret 1553, p. 15 ; et La Porte, Les Epithetes, s.v. «Aiguillon», "Amoureus ou Aimant » et nombreuses occurrences de l'épithète amoureus dans le relevé, passim. 51. Ronsard, Lm, t. IV, p. 8, v. 3 ; Muret 1553, p. 17 ; et La Porte, Les Epithetes, s.v. « Dard ».

52. Ronsard, Lm, t. IV, p. 19, v. 12 ; Muret 1553, p. 30 ; et La Porte, Les Epithetes, s.v. «Bellerophon ».

53. Ronsard, Lm, t. IV, p. 33, v. 2 ; Muret 1553, p. 51 ; et La Porte, Les Epithetes, s.v. "Ange», comm. (cité en figure 9).

54. La Porte reconnaît d'ailleurs sa dette dans son "Advertissement au lecteur », Les Epithetes, f. ã iij $r^{\circ}$.

55. Voir A.-P. Pouey-Mounou, « La Porte et les commentaires des Amours », art. cit.

56. Muret 1553, p. 19, et La Porte, Les Epithetes, s.v. « Chorebe».

57. Muret 1553, p. 71-72, et La Porte, Les Epithetes, s.v. « Ixion » et « Tantale ». La précision selon laquelle Tantale fut «roi de Phrygie», origine probable de l'épithète phrygien, figure dans le commentaire de La Porte mais ne vient pas de Muret.

58. Muret 1553, p. 97 (v. 3, un tans pluvieus), et La Porte, Les Epithetes, s.v. « Arc-en-ciel ». Voir A.P. Pouey-Mounou, « La Porte et les commentaires des Amours », art. cit. 
59. Pour d'autres exemples non ronsardiens de cette pratique de La Porte, voir id., "Brocards, pastiches et mélanges: bas morceaux choisis des Epithetes de La Porte", Textes au corps. Promenades et musardises sur les terres de Marie Madeleine Fontaine, Genève, Droz, 2015, p. 129-145, notamment p. 130-135, à partir d'exemples de Marot et de Du Bellay.

60. Muret 1553, p. 79, et La Porte, Les Epithetes, s.v. « Castor » et « Pollux ».

61. Ronsard, Lm, t. VIII, p. 319, v. 579-581: «O fameux Escuyers, Cavalcadours, Guerriers, / Escrimeurs, Voltigeurs, Soldatz, \& Mariniers, / O les filz putatifz du Spartain Tyndarée ».

62. Muret 1553, p. 51, v. 2, p. 52, v. 3 et 5, p. 180, v. 2, et p. 201, v. 1.

63. Ronsard, Lm, t. VIII : Hymne de la Justice (p. 47-72), Hymne de la Philosophie (p. 85-102), Les Daimons (p. 115-139), Hymne du Ciel (p. 140-149), Hymne des Astres (p. 150-161), Hymne de la Mort (p. 161-179), Hymne de l'Or (p. 179-205), Hymne de l'Éternité (p. 246-254).

64. Exactement : 1136 substantifs et 1645 épithètes.

65. Le balisage du corpus tel qu'il est présenté en figures 10 et 12 n'est que partiellement restitué : les éléments des ponctuations ont été supprimés partout, seules les ponctuations sont présentes, et tous les attributs des éléments qui balisent les épithètes et les attributs ne sont pas toujours mentionnés (par exemple, les @xml :id sont en figure 10 mais non en 12).

66. Voir A.-P. Pouey-Mounou et N. Gasiglia, «Le lexicographe au travail. Le traitement des Hymnes cosmologiques de Ronsard (1555-1556) dans les Epithetes de La Porte ", L'épithète, la rime et la raison, op. cit., p. 237-277.

67. Exactement : 1656.

68. Sur le statut rhétorique de telles formules, voir N. Laurent, «Les rapports entre le nom propre et le nom commun dans la réflexion sur l'antonomase aux $\mathrm{XVI}^{\mathrm{e}}$ et $\mathrm{XVII}^{\mathrm{e}}$ siècles ", Le Français préclassique (1500-1650), 16, 2014, p. 67-79.

69. Sur tous ces exemples, voir A.-P. Pouey-Mounou et N. Gasiglia, «Le lexicographe au travail... ", art. cit., p. 247-262.

70. Ronsard, Hymne de la Philosophie, Lm, t. VIII, p. 94, v. 147.

71. Ronsard, Hymne du Ciel, Lm, t. VIII, p. 146, v. 78.

72. Ronsard, Hymne de l'Éternité, Lm, t. VIII, p. 250, v. 73.

73. Cette manipulation a été présentée dans le cadre d'une session de l'«Atelier $x v I^{\mathrm{e}}$ siècle » de M. Huchon sur Les Qualifications des dieux, org. A.-P. Pouey-Mounou, 18 oct. 2014, Paris-Sorbonne.

74. Avec réfection étymologique dans le cas de Satyre. Cf. A. Rey, Dictionnaire historique de la langue française, Paris, Le Robert, 1992, s.v.

75. Si nous en venions à constituer un corpus électronique de référence des textes de la Pléiade dépouillés par La Porte, ce qui n'est pas actuellement envisagé, le choix qui a été fait de fusionner les citations courtes référencées et analysées au texte dictionnairique serait peut-être révisé en fonction de ceux qui seraient faits pour permettre un accès aux citations longues du corpus à partir de l'interface dictionnairique. En effet, les citations longues ne méritant pas d'être incluses aux côtés des citations courtes dans les articles (les répétitions des textes ou de leurs extraits longs seraient absurdes), il faudrait opter pour une annotation déportée, qui permette aux citations courtes ou aux épithètes sous lesquelles elles sont enregistrées de pointer vers les éléments XML enchâssant les citations longues dans le corpus. En outre, nous pourrions alors imaginer une interface de lecture des textes du corpus et un accès au dictionnaire par hyperappel, à partir des substantifs et des épithètes qu'ils contiennent. Le dictionnaire enrichi des citations courtes analysées ne serait plus alors un produit éditorial autonome mais un constituant d'un environnement numérique offrant, de manière articulée, la consultation de textes de la Pléiade (le corpus) et des Epithetes.

76. Voir N. Gasiglia et A.-P. Pouey-Mounou, «La Porte, la Pléiade et Bacchus », Inqualifiables fureurs. Les qualifications des figures de l'inspiration à la Renaissance, éd. M. Huchon et A.-P. PoueyMounou, Paris, Classiques Garnier, à paraître. Sur l'histoire éditoriale des Folastries, voir Ronsard, éd. Pléiade, t. I, p. 1448-1449. 
77. Ronsard, Lm, t. III, p. 184-217, et Magny, Les Martinales, dans Les Gayetez [1554], éd. A. R. Mac Kay, Genève, Droz, p. 74-89.

78. Magny, Les Martinales, éd. cit., p. 87, v. 372, et p. 86, v. 325.

79. Ronsard, Lm, t. III, p. 199, v. 271 (Thebain père), et p. 207, v. 438 (Père Evien).

80. Ronsard, Lm, t. V, p. 65, v. 183 (ce Père Bromien), et p. 60, v. 100 (comme un Roy vainqueur).

81. Ronsard, Lm, t. V, p. 53-76, et Baï, Poemes, IV, 11, dans Euvres complètes, I, Euvres en rime, I ${ }^{\text {re }}$ partie, Neuf Livres des Poemes, éd. J. Vignes (dir.) et al., Paris, Champion, p. 294-299. Voir la notice de cette pièce, ibid., p. 776-780.

82. Le rompt-souci de Ronsard (Lm, t. V, p. 59, v. 82) prévaut, notamment, dans ses relevés sur le dieu brise-souci de Baïf (éd. cit., p. 298, v. 165). Quant au terme daimon (Baï, p. 299, v. 186, Daimon aime-dance), il est aussi dans les Dithyrambes de Ronsard (Lm, t. V, p. 74, v. 355, O Daimon) et peut avoir été médiatisé par ce texte.

83. Marulle, Baccho, dans Hymnes naturels, éd.-trad. J. Chomarat, Genève, Droz, 1995, I, 6, p. 64-76. Voir les annotations d'A. Gendre (Ronsard, Les Amours et Les Folastries (1552-1560), éd. A. Gendre, Paris, LGF, 1993, p. 285-304).

84. Ronsard, Lm, t. VI, p. 176-190.

85. C'est ce que souligne en 1604 son commentateur Besly (Les CEuvres de P. de Ronsard, Paris, N. Buon, 1604, 10 t. en 5 vol. , t. VII, p. 263) en commentant le fait que Dorat, traducteur en latin de l'Hinne de Bacus (Paris, A. Wechel, 1555), n'a guère utilisé Marulle (à l'exception de l'épithète solivagus : Marulle, Baccho, éd. cit., v. 26).

86. Baif, éd. cit., p. 298-299, v. 166-167 (O Nictelien / O Semelien) et 184-185 (O Semelien, O Nictelien); Ronsard, Lm, t. V, p. 69, v. 273 (Nyctelien); et La Porte, Les Epithetes, s.v. « Bacchus » (nictolien).

87. Il s'agit des épithètes joyeux (mais la périphrase le bon Pere joyeux est déjà présente dans la Folastrie VIII, Lm, t. V, p. 51, v. 92), deux fois né, ainsi que libre (pour Liber) et vainqueur indien (périphrase roy des Indes vainqueur, assez éloignée).

88. Trésor de la langue française. Dictionnaire de la langue du XIX ${ }^{e}$ et du XX ${ }^{e}$ siècle (1789-1960), 16 vol. , Paris, Éditions du Centre National de la Recherche Scientifique (t. 1-10) / Gallimard (t. 11-16), 1971-1994.

\section{RÉSUMÉS}

L'article analyse les contraintes et les choix méthodologiques qu'implique le traitement d'un corpus lexicographique ancien, stylistiquement exploitable, à partir de l'exemple d'un projet éditorial en cours : le traitement et l'édition numérique d'un recueil lexicographique du XVI ${ }^{\mathrm{e}}$ siècle, Les Epithetes de Maurice de La Porte (1571), qui a pour source principale la poésie de la Pléiade. La base textuelle en cours de constitution, qui doit permettre de repérer les attestations à partir desquelles La Porte a effectué ses sélections, se veut à la fois un outil d'analyse et l'état préparatoire d'une édition numérique, dotés de fonctionnalités de recherche originales fondées sur l'annotation des attestations dont le texte de La Porte aura été enrichi. Après avoir souligné la spécificité de ce recueil, en tant que dictionnaire de cooccurrences, dans la production lexicographique actuelle et au XVI ${ }^{\mathrm{e}}$ siècle, l'article développe les contraintes inhérentes à ce corpus, des contraintes qui sont liées à son objet rhétorique autant qu'à l'état de la langue française de cette époque et à l'interprétation du lexicographe ; il revient enfin sur ces difficultés 
à travers deux exemples concrets de manipulations de corpus, afin de présenter le potentiel informationnel de ces données et les stratégies manipulatoires envisagées.

This article analyses the constraints and methodological choices involved in processing a premodern lexicographical corpus that is stylistically usable. It is based on the example of a current editorial project that involves processing and publishing, in digital format, a sixteenth century lexicographical work, Les Epithetes by Maurice de La Porte (1571), whose main source is the poetry of the Pléiade. The textual database currently being established should make it possible to identify the attestations upon which La Porte based his selections. The database is intended to be both an analytical tool and the preparatory phase for a digital edition of the work. It features original search functions based on the annotation of the attestations that will enrich La Porte's text. The article underlines the specificity of this dictionary of collocations in modern and sixteenth century lexicographical output, and discusses the constraints inherent in this particular corpus. These relate to its rhetorical purpose, as well as to the state of the French language at the time and the interpretation of the lexicographer. The article illustrates these constraints via two concrete examples of how corpora can be used, so as to present the informative potential of the data and the strategies that can be implemented to exploit it.

INDEX

Mots-clés : corpus électronique ; lexicographie ; dictionnaire de cooccurrences

Keywords : electronic corpus ; lexicography ; dictionaries of collocations

\section{AUTEURS}

NATHALIE GASIGLIA

UMR 8163 (STL), Université de Lille ; nathalie.gasiglia@univ-lille3.fr

\section{ANNE-PASCALE POUEY-MOUNOU}

EA 4509 (STIH), Université Paris-Sorbonne / IUF ; ap.pouey@free.fr 